\title{
The global gene expression outline of the bovine blastocyst: reflector of environmental conditions and predictor of developmental capacity
}

Dessie Salilew-Wondim', Dawit Tesfaye ${ }^{2}$, Franca Rings ${ }^{1}$, Eva Held-Hoelker ${ }^{1}$, Dennis Miskel ${ }^{1}$, Marc-Andre Sirard ${ }^{3}$, Ernst Tholen ${ }^{1}$, Karl Schellander ${ }^{1}$ and Michael Hoelker ${ }^{1,4^{*}}$

\begin{abstract}
Background: Morphological evaluation of embryos has been used to screen embryos for transfer. However, the repeatability and accuracy of this method remains low. Thus, evaluation of an embryo's gene expression signature with respect to its developmental capacity could provide new opportunities for embryo selection. Since the gene expression outline of an embryo is considered as an aggregate of its intrinsic characteristics and culture conditions, we have compared transcriptome profiles of in vivo and in vitro derived blastocysts in relation to pregnancy outcome to unravel the discrete effects of developmental competence and environmental conditions on bovine embryo gene expression outlines. To understand whether the gene expression patterns could be associated with blastocyst developmental competency, the global transcriptome profile of in vivo (CVO) and in vitro (CVT) derived competent blastocysts that resulted in pregnancy was investigated relative to that of in vivo (NVO) and in vitro (NVT) derived blastocysts which did not establish initial pregnancy, respectively while to unravel the effects of culture condition on the transcriptome profile of embryos, the transcriptional activity of the CVO group was compared to the CVT group and the NVO group was compared to the NVT ones.
\end{abstract}

Results: A total of 700 differentially expressed genes (DEGs) were identified between CVO and NVO blastocysts. These gene transcripts represent constitutive regions, indel variants, 3'-UTR sequence variants and novel transcript regions. The majority (82\%) of these DEGs, including gene clusters like ATP synthases, eukaryotic translation initiation factors, ribosomal proteins, mitochondrial ribosomal proteins, NADH dehydrogenase and cytochrome c oxidase subunits were enriched in the CVO group. These DEGs were involved in pathways associated with glycolysis/glycogenesis, citrate acid cycle, pyruvate metabolism and oxidative phosphorylation. Similarly, a total of 218 genes were differentially expressed between CVT and NVT groups. Of these, 89\%, including TPT1, PDIA6, HSP9OAA1 and CALM, were downregulated in the CVT group and those DEGs were overrepresented in pathways related to protein processing, endoplasmic reticulum, spliceasome, ubiquitone mediated proteolysis and steroid

\footnotetext{
* Correspondence: michael.hoelker@uni-goettingen.de

'Institute of Animal Sciences, Animal Breeding, University of Bonn, Endenicher Allee 15, 53115 Bonn, Germany

${ }^{4}$ Department of Animal Science, Biotechnology \& Reproduction in farm animals, University of Goettingen, Burckhardtweg 2, 37077 Goettingen, Germany

Full list of author information is available at the end of the article
}

(c) The Author(s). 2021 Open Access This article is licensed under a Creative Commons Attribution 4.0 International License, which permits use, sharing, adaptation, distribution and reproduction in any medium or format, as long as you give appropriate credit to the original author(s) and the source, provide a link to the Creative Commons licence, and indicate if changes were made. The images or other third party material in this article are included in the article's Creative Commons licence, unless indicated otherwise in a credit line to the material. If material is not included in the article's Creative Commons licence and your intended use is not permitted by statutory regulation or exceeds the permitted use, you will need to obtain permission directly from the copyright holder. To view a copy of this licence, visit http://creativecommons.org/licenses/by/4.0/ The Creative Commons Public Domain Dedication waiver (http://creativecommons.org/publicdomain/zero/1.0/) applies to the data made available in this article, unless otherwise stated in a credit line to the data. 


\begin{abstract}
biosynthesis. On the other hand, although both the CVT and CVO blastocyst groups resulted in pregnancy, a total of 937 genes were differential expressed between the two groups. Compared to CVO embryos, the CVT ones exhibited downregulation of gene clusters including ribosomal proteins, mitochondrial ribosomal protein, eukaryotic translation initiation factors, ATP synthases, NADH dehydrogenase and cytochrome c oxidases. Nonetheless, downregulation of these genes could be associated with pre and postnatal abnormalities observed after transfer of in vitro embryos.

Conclusion: The present study provides a detailed inventory of differentially expressed gene signatures and pathways specifically reflective of the developmental environment and future developmental capacities of bovine embryos suggesting that transcriptome activity observed in blastocysts could be indicative of further pregnancy success but also adaptation to culture environment.
\end{abstract}

Keywords: Bovine, Embryo, Transcriptome, Pregnancy

\section{Introduction}

Selecting transferable embryos that could sustain pregnancy has been a challenge in the field of assisted reproductive technology. Indeed, in humans, non-invasive selection strategies based on morphological evaluation have been used to select the best embryos. These grading techniques take into account the appearance of the cytoplasm, size and shape of blastomeres, embryo fragmentation [1], number of cleavages (even or uneven) [1, 2], cleavage kinetics [3], blastomere multinucleation [47] or a combination of these [8]. Morphological classification of bovine embryos prior to transfer to recipient animals represents the common practice. Usually, the bovine embryo is morphologically classified as grade 1 (excellent or good), grade 2 (regular), grade 3 (poor) or grade 4 (dead or degenerating embryos). Grade 1 in vivo derived embryos are eligible for international trade as they are suggested to be viable and to survive freeze/ thawing well, whereas grade 2 and 3 are recommended for transfer fresh into recipient animals [9]. Although, morphological classification methods have substantial value, repeatability and accuracy of morphological parameters are generally fraught with errors due to the subjectivity of classification. Moreover, even embryos graded as low quality might be able to develop to term [10] suggesting that selecting embryos based on morphological appearance has potential drawbacks. Furthermore, preimplantation genetic screening by testing for chromosomal abnormalities as well as activity of genes related to metabolism has been used for selecting developmentally competent embryos [11]. Therefore, an embryo screening method that provides complete information about an embryo's intrinsic characteristics, such as its metabolism and its gene expression pattern could be an alternative to subjective analysis during selection. In that regard, it would be interesting to identify and characterize molecular signatures that are associated with an embryo's developmental capacity. For instance, understanding the expression of genes that could cause termination of pregnancy by affecting embryonic genome activation, blastocyst formation, embryo elongation or secretion of interferon-tau [12] could be one step forward to identify molecular markers useful for classifying an embryo's individual developmental potential. This, however, could be even more relevant for in vitro derived embryos since their developmental capacity might be more compromised by its non-physiological preimplantation environment factors like culture media, in vitro culture conditions such as oxygen level, $\mathrm{pH}$, temperature, humidity and others. Indeed, oxygen tension [13], heat stress [14] as well as the principal formulation of the culture medium itself $[15,16]$ were found to affect the developmental competence of bovine embryos by altering the expression of genes associated with pluripotency, trophectoderm formation and apoptosis. Subsequently, suboptimal in vitro culture conditions are suggested to hinder embryonic developmental competence by altering the expression profile or epigenetic landscape of genes associated with embryonic development. With this respect, a previous study has shown alterations in expression of 134 transcripts at 4-cell stage and 97 transcripts in 8-cell stage embryos derived from in vitro compared to the in vivo derived ones [17], indicating the impact of the in vitro culture environment on the gene expression patterns of the bovine embryo. Similarly, a stage specific exposure of bovine embryos to in vitro culture condition before or after embryonic genome activation has unravelled alterations in expression of genes involved in lipid metabolism and oxidative phosphorylation [18]. Moreover, several candidate genes and large scale transcriptome profile analysis approaches [18-24] and DNA methylation studies [25-30] have proven the effect of culture conditions on gene expression patterns and epigenetic profiles in the resultant blastocysts. Collectively, it is generally accepted that the transcriptome profile of the bovine blastocyst depends on the culture conditions of the in vitro culture. However, all studies comparing the gene expression signature 
of bovine embryos derived from different culture environments so far have not considered the developmental capacity of the individual embryos analysed. With this respect, it is well known that in vivo derived embryos develop to a much higher extent into healthy offspring compared to in vitro derived ones, it is questionable to compare populations of in vivo and in vitro derived embryos containing contrasting proportions of embryos bearing high and low developmental capacity. Therefore, doing so indicates not only the effect of the environment but also the impact of contrasting proportions of competent embryos analysed in these studies. Thus, the consequence of contrasting intrinsic embryo qualities has been measured and interpreted wrongly as reflecting the environment.

The outline of an embryo's transcriptome profile could be used to predict the embryo's individual developmental capacity. This is based on the hypothesis that, unlike the non-competent ones, competent in vivo or in vitro derived developmentally competent embryos are endowed with typical molecular signatures necessary to support further development. Thus, investigating the association between embryonic developmental competence and their molecular signatures could provide an opportunity to generate molecular markers that could be used as predictors of embryonic developmental competence. Earlier, we and others have demonstrated the correlation between gene expression patterns of in vitro or in vivo produced bovine blastocyst with their developmental competence [31-35]. However, for some studies [3133], the numbers of probes (including the controls) incorporated in the microarray platform were few. Other studies, [34, 35] were focused on the gene expression patterns in relation to female embryo developmental competency. Moreover, previous conclusions drawn about the gene expression of developmentally competent in vivo and in vitro derived bovine embryos was done indirectly by performing meta-analysis, but no direct comparison between competent embryos derived from different developmental environments has been conducted so far. Thus, molecular signatures which are predictors of developmental capacity without interfering effects of the given developmental environment have not been specifically determined so far. Therefore, further studies correlating the gene expression signature with future developmental capacity are necessary to further enhance our predictive power in determining the developmental capacity of bovine embryos.

Collectively, it is unquestionable that the gene expression outline of the bovine embryo partially reflects its culture environment during early development as well as predicting its future developmental capacity. The latter in turn, might be partially affected by the culture environment as well as predetermined by the embryo's intrinsic quality independent from the culture environment. Therefore, the principal aim of the present study was first to unravel specific molecular signatures predictive for developmental capacity and to identify those molecules specifically reflective of culture/environmental conditions. These insights might be beneficial for selecting the best embryo for transfer but also to unravel environmental conditions interactively affecting the expression outline of genes related to viability.

\section{Materials and methods}

\section{Experimental design}

To unravel the proposed questions of the present study, four gene expression studies were conducted (Fig. 1). To unravel the gene expression patterns specifically caused by contrasting developmental capacities which are typical for the in vivo derived embryos, I) the transcriptome profile of competent in vivo derived embryos (CVO) was compared with the profile of non-competent in vivo derived embryos (NVO). Likewise, to unravel the gene expression signature specifically caused by contrasting developmental capacities typical for vitro derived embryos, II) the transcriptome profile of competent in vitro derived embryos (CVO) was compared with the profile of non-competent in vitro derived embryos (NVO). To explore the gene expression pattern of bovine embryos being specifically a consequence of contrasting culture conditions without conflictive with developmental capacity, III) the transcriptome profile of competent in vitro derived embryos (CVT) was compared with the profile of competent in vivo derived embryos (CVO). Finally, to explore the gene expression pattern of bovine embryos caused by contrasting culture conditions that conflict with developmental capacity, IV) the gene expression profile of non-competent in vitro derived embryos (NVT) was compared with non-competent in vivo derived embryos (NVO) ones.

\section{Animal handling}

Animal handling for collection of in vivo derived embryos and transfer of both in vivo and in vitro derived embryos to synchronized recipients was carried out in accordance with the German Law of Protection (TierSchG \& TierSchVersV). All experimental protocols performed on cows in this study were approved by the state office for Nature, Environment, and Consumer Protection of North Rhine-Westphalia, Germany (Landesamt für Natur, Umwelt und Verbraucherschutz Nordrhein-Westfalen, Deutschland) under license number 84-02.04.2014.A499. All experiments were performed in accordance with relevant guidelines and regulations and adheres to the ARRIVE guidelines. 


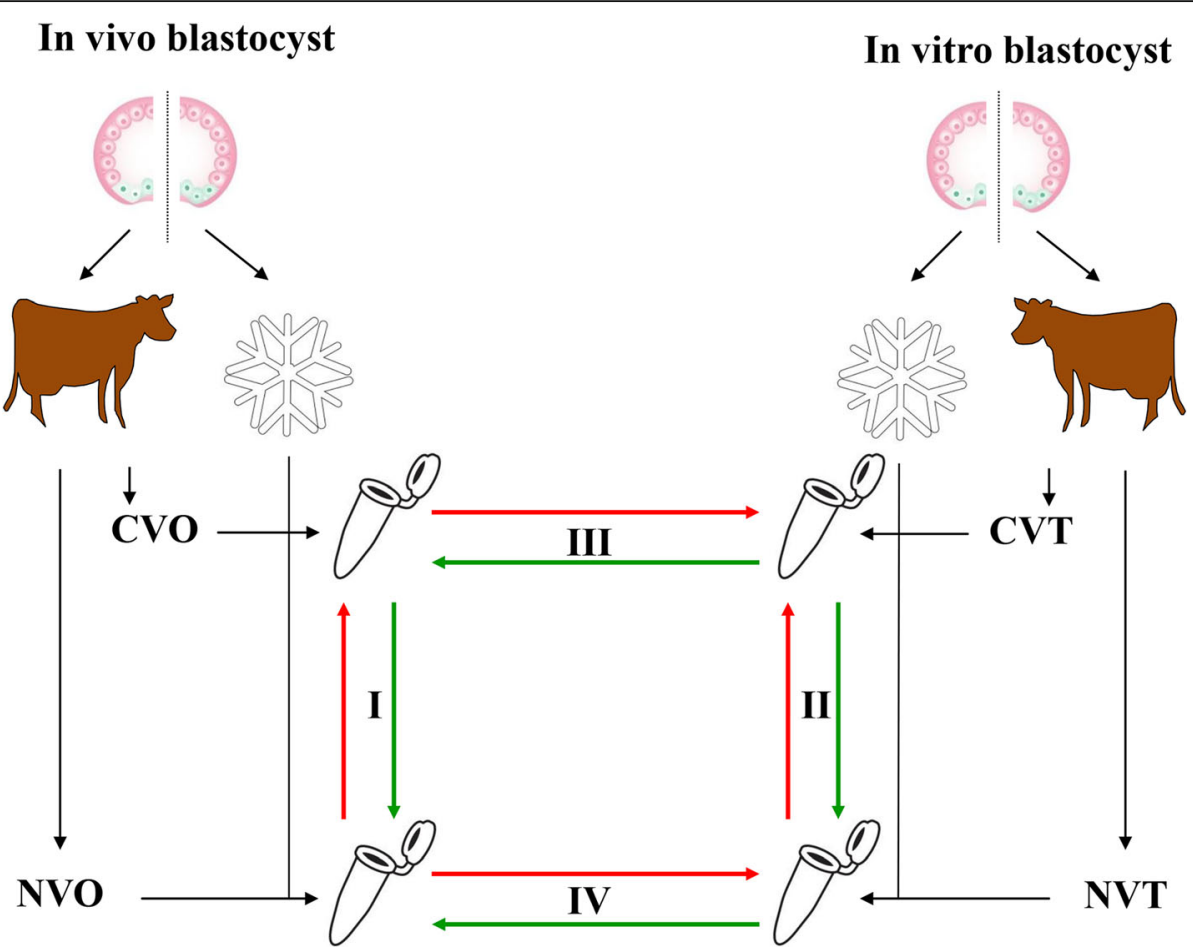

Fig. 1 The experimental design used for comparative gene expression analysis in embryo biopsies. Numbers I, II, III and VI indicate comparisons with regard to the global gene expression profile between competent (CVO) and non-competent (NVO) in vivo derived blastocysts, competent $(\mathrm{CVT})$ and non-competent (NVT) in vitro derived blastocysts, competent in vitro derived blastocysts (CVT) and competent in vivo derived blastocysts (CVO) as well as non-competent in vitro derived blastocysts (NVT) and non-competent in vivo derived blastocysts (NVO), respectively

\section{In vitro embryo production (IVP)}

In vitro embryo production was performed using oocytes collected from abattoir-derived ovaries. The bovine ovaries were transported to the laboratory (Campus Frankenforst) of the University of Bonn in a thermo flask containing $0.9 \%(\mathrm{w} / \mathrm{v})$ saline solution. Upon arrival, the ovaries were washed three times with $0.9 \%$ saline solution. Afterwards, cumulus-oocyte complexes (COCs) were aspirated from 2 - to $8 \mathrm{~mm}$-diameter follicles. The COCs were then in vitro matured, in vitro fertilized and in vitro cultured as indicated previously [31]. The developmental rates were recorded, and day 7 blastocysts were collected for transfer.

\section{In vivo embryo production}

Simmental heifers were used in in vivo embryo production and embryo transfer. All experimental animals were handled and managed according to the rules and regulations of the German law of animal protection at Campus Frankenforst of the University of Bonn. The procedures of in vivo embryo production was performed as indicated previously [32, 33]. Briefly, Simmental heifers were pre-synchronized using intramuscular administration of $500 \mathrm{mg}$ of the prostaglandin $\mathrm{F}_{2 \alpha}(\mathrm{PGF} 2 \alpha)$ analogue cloprostenol (Estrumate; Munich, Germany) twice within
11 days and followed by $0.02 \mathrm{mg} \mathrm{GnRH}$-analogue buserelin (Intervet, Boxmeer, The Netherlands) administration after 2 days of each of PGF2a administration. Twelve days after the second GnRH administration, 8 consecutive FSH-injections over 4days in decreasing doses was performed followed by two $\mathrm{PGF}_{2 \alpha}$ treatments, 60 and $72 \mathrm{~h}$ after the initial FSH. Ovulation was induced by administration of $0.02 \mathrm{mg}$ buserelin and was followed by three inseminations at $12 \mathrm{~h}$ intervals. Embryos were flushed 7 days after insemination.

\section{Blastocyst biopsy transfer}

Blastocyst biopsying and transfer was performed as reported previously [31, 32]. Briefly, a 40-50\% portion containing both inner cell mass (ICM) and trophectoderm (TE) was biopsied from each blastocyst and snap frozen in cryo-tubes, containing lysis buffer [0.8\% Igepal (Sigma-Aldrich, MO, USA), $40 \mathrm{U} \mathrm{ml}^{-1}$ RNasin (Promega, WI, USA), $5 \mathrm{mM}$ dithiothreitol) for further analysis. The remaining $50-60 \%$ portion of each blastocyst was in vitro cultured in Charles Rosenkrans 1 medium supplemented with amino acid for $2 \mathrm{~h}$ and transferred to synchronized Simmental heifers. A total of 69 in vivo derived and 59 in vitro derived blastocyst biopsies were transferred to 128 recipient heifers. 
Pregnancy diagnosis and embryo biopsy categorization Pregnancy diagnosis was performed on days 28 and 42 using ultrasonography (Pie Medical, $5 \mathrm{MHz}$ ) and at day 90 by rectal palpation. Following this, in vivo and in vitro derived embryo biopsies taken from those blastocysts which sustained pregnancy until 90 days of gestation were classified as competent in vivo derived (CVO) and competent in vitro derived (CVT) blastocysts, respectively. Similarly, embryo biopsies taken from those blastocysts which did not initiate initial pregnancy were classified as non-competent in vivo derived (NVO) and non-competent in vitro derived (NVT) blastocysts, respectively.

\section{RNA isolation from embryo biopsies}

Total RNAs was isolated from each blastocyst biopsy group (CVO, CVT, NVO, and NVT) in four independent replicates. Each replicate consists of 5 biopsies and RNA isolation was performed using the PicoPure RNA isolation kit (Arcturs, Munich, Germany) following the manufacturer's protocol. Briefly, each embryo biopsy was incubated with $20 \mu \mathrm{l}$ extraction buffer at $42^{\circ}$ for 30 min. Biopsies from the same group were pooled. After adding 1 volume $70 \%$ ethanol, the samples were loaded into the pre- conditioned purification column. The RNA was bound to the column by centrifugation of the samples at $1057 \mathrm{rpm}$ for $2 \mathrm{~min}$, followed by a centrifugation step at $13500 \mathrm{rpm}$ for $30 \mathrm{~s}$. The samples were washed using wash buffers and on column DNase treatment was performed using RNase-fee DNase I (Qiagen, CA, USA). After subsequent steps, the RNA was eluted in $12 \mu$ l elution buffers. The quality and concentration of RNA and was evaluated using NanoDrop 8000 Spectrophotometer.

\section{RNA amplification and array hybridization}

The total RNA samples were subjected two rounds of amplification to generate amplified anti-sense RNA using the RNA amplification HS kit (Applied Biosystems). The amplified RNA was eluted in $30 \mu \mathrm{l}$ of elution buffer and the quality and quantity of amplified RNA samples was evaluated using the NanoDrop 8000 Spectrophotometer. Two microgram of amplified RNA from each sample (CVO, CVT, NVO and NVT) was mixed with either $1 \mu \mathrm{l}$ of $\mathrm{Cy}-3$ or $\mathrm{Cy}-5$ ULS fluorescent labelling kit (Kreatech Diagnostics, Amsterdam, Netherlands) and incubated at $85^{\circ} \mathrm{C}$ for $30 \mathrm{~min}$. Unincorporated $\mathrm{Cy}-3$ and $\mathrm{Cy}-5$ dyes were removed using the PicoPure RNA extraction kit (Applied Biosystems). Following this, sample mixing was performed following the outline of the experimental design (Fig. 1). Samples were mixed with $157.6 \mu \mathrm{l}$ hybridization cocktail and incubated at $95^{\circ} \mathrm{C}$ for $3 \mathrm{~min}$ and at $37^{\circ} \mathrm{C}$ for $30 \mathrm{~min}$. Afterwards, $65 \mu \mathrm{l}$ of Agilent-CGHBlock was added to each sample and transferred onto the EmbryoGENE bovine microarray slides. Each slide contains four arrays, and each array consists of 45,000 probes. The slides were then incubated for $40 \mathrm{~h}$ at $65^{\circ} \mathrm{C}$ in the hybridization oven. At the end of hybridization, the slides were sequentially washed for $10 \mathrm{~min}$ in $2 \mathrm{x}$ SSC plus $0.1 \%$ SDS, $5 \mathrm{~min}$ each in $0.2 \mathrm{xSSC}$ and $0.1 \%$ SSC buffers, $1 \mathrm{~min}$ each in water and isopropanol. Array hybridization was done in a dye-swap design (technical replicates) and for each sample three independent replicates were performed. A total of 24 hybridizations were done for 4 experiments.

\section{Array image capture and array data analysis}

The arrays were scanned using Axon GenePix 4000B scanner and the images of the array were analysed using GenePix Pro analysis software (version 5.0) (Axon Instruments, Foster City, CA) as indicated previously [32]. Briefly, subtract and offset method was used to correct the array background [36] and LOESS and scale-normalization methods were used to normalize differences within array variations [37, 38] and between the arrays, respectively. A mean $\log _{2}$ transformed value of (Cy5/Cy3) was calculated from three replicates and the respective dye-swaps to obtain one value per target. Differentially expressed genes were identified using linear models for microarray data [39]. Genes with average $\log _{2}$ expression value $\geq 0.65$ and $\leq-0.65$ fold change and $p<0.05$ and adjusted $p$ value $($ FDR $)<0.2$ were considered as differentially expressed genes.

\section{Results}

A total of 59 biopsied in vitro derived and 69 biopsied in vivo derived embryos were transferred to synchronized Simmental heifers. Of these, 17 (25.4\%) biopsied in vitro derived embryos ended up in a stable pregnancy at day 90. Similarly, 15 (24.6\%) biopsied in vivo derived embryos were ended up in a stable pregnancy at day 90 . Embryos that did not end up in pregnancy at day 28 days of gestation were classified as non-competent embryos and those which resulted in pregnancy at least until day 90 of the gestation period were classified as developmentally competent. To get insight into specific differences with regard to the gene expression outline caused by contrasting viabilities (competent vs. non-competent blastocysts derived from the same environment) as well as caused specifically by contrasting environments (in vitro vs. in vivo derived embryos of equal developmental capacity), the blastocyst biopsies were classified based on pregnancy outcome of the corresponding counterparts as competent in vivo blastocyst (CVO), non-competent in vivo blastocyst (NVO), competent in vitro blastocyst (CVT) and non-competent in vitro blastocyst (NVT). 
Molecular signatures predicting the developmental capacity of in vivo derived bovine embryos

A total of 766 probes associated with 700 gene transcripts were identified to be differentially expressed between competent in vivo derived embryos (CVO) and non-competent in vivo derived embryos (NVO), (Fig. 2, Table 1). The expression pattern of 634 differentially expressed genes (DEGs) including RPL34, RPS28, RPS24, KRT19, GLRXL and SERBP1 transcripts were significantly increased whereas expression of 132 gene transcripts including NANOG, CYP51A1, TNIP2, BCAT2, FOSL1 and ACTB was significantly decreased in embryos of the CVO group (Fig. 2, Supplemental Table S1). Since the EmbryoGENE microarray (Agilent-028298: Bovine Embryo and Splice Transcriptome microarray) is enriched with annotated genes, uncharacterized transcribed regions, embryo specific indel type variants, alternative 3'UTR events (genes) and pseudo genes [40], we took advantage of this opportunity to investigate the proportion of these gene expression features. Accordingly, a total of 21 (0.06\%) and 188 (24.4\%) DEGs represented pseudo genes and novel transcripts (NTRs), respectively (Table 1) and a total of 29 genes (3.8\% of all DEGs) including ALDH3A2, TFAP2C, UBE3B, NET1, SNX16, SLC35E3, MAML2, CDYL and CYP51A1 represented alternative 3 -'UTR events (Tale 1, Table 2). Similarly, a total of 44 differential expressed transcripts including PLAC8, PRDX5, MYL7 and MYL6 represented gene variants (Table 3). Strikingly, the expression trends of six out of ten MYL6 variants were upregulated in embryos of CVO compared to the NVO group.

Expression of gene cluster predicting the developmental capacity of in vivo derived bovine embryos

In this study, we identified several gene cluster, each comprising of a group of genes potentially sharing a generalized function, exhibiting higher expression in CVO compared to NVO samples, that predict developmental capacity of in vivo derived bovine embryos. These gene cluster are mainly associated with mitochondrial functions and include ATP synthases (ATP5E, -G1, -G2, $-H,-I,-J,-J 2,-L,-O)$, eukaryotic translation initiation factors (EIF1, $-3 C$, EIF3D, $-E,-K, E I F 4 E 2)$, ribosomal proteins $(R P L 7,-11,-12,-13 .-15,-23,-24,-27 A$, $-30,-31,-34,-35 A,-36,-37 A,-38,-39$, RPS3, -6 , $-8,-21,-24,-28)$, mitochondrial ribosomal proteins, NADH dehydrogenases (NDUFS1, $-2,-4,-5,-8$, NDUFB8), cytochrome c oxidases, aldehyde dehydrogenases, proteasomes, WD repeats and keratins (Fig. 3). Higher expression of these gene clusters specifically in the competent in vivo derived embryos $(\mathrm{CVO})$ could indicate the upregulation of global protein translation turnover and ATP generating pathways.

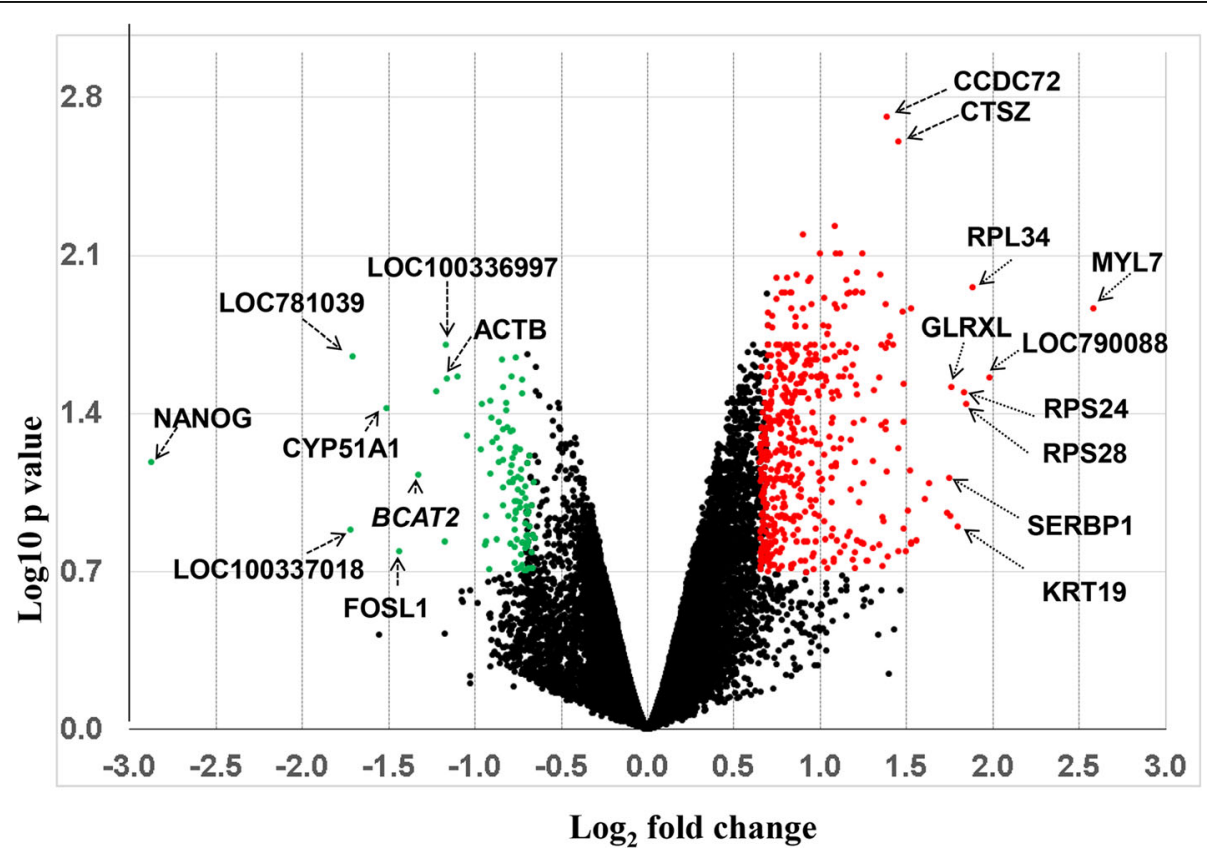

Fig. 2 Molecular signature associated with the developmental capacity of in vivo derived embryos. Volcano plot demonstrating differentially expressed genes between CVO and NVO blastocysts. Red and green dots indicate up and downregulated genes, respectively in CVO compared to NVO blastocysts. Transcripts highly significant up or downregulated are indicated with arrows 
Table 1 Preferentially expressed probes in CVO and NVO groups

\begin{tabular}{llll}
\hline & Enriched in CVO & Enriched in NVO & Total \\
\hline Constitutive & 422 & 74 & 506 \\
Novel gene transcribed regions; evidence: embryonic ESTs & 158 & 30 & 188 \\
Alternative 3'UTR events (genes) & 10 & 19 & 29 \\
Indel type splice variants & 35 & 9 & 44 \\
Pseudo genes & 23 & 3 & 26 \\
Total differentially expressed probes & 635 & 131 & 766 \\
\hline
\end{tabular}

\section{Molecular signatures predicting the developmental capacity of in vitro derived bovine embryos}

The gene expression analysis in the competent in vitro derived embryos (CVT) and non-competent in vitro derived embryos (NVT) at blastocyst stage showed a differential expression of 218 gene transcripts (226 probes) (Table 4, Supplemental Table S2). Of these, the expression of 194 genes was increased while expression of 24 genes was decreased in CVT compared to NVT samples. Among the DEGs, TPT1, PDIA6, HSP90AA1 and CALM were among the top upregulated genes demonstrating differential expression by $3.5-4.5$ folds $(p<0.05)$ whereas STAT1, OTUB1, EIF1AD and EGLN1 were among the top downregulated genes (23-35 folds) in CVT compared to NVT group (Fig. 4). Moreover, about 1.8 and $8.4 \%$ of all DEGs represented splice variants (Table 5) and alternative 3 '-UTR events (Table 6), respectively. However, the total number of DEGs between CVT vs. NVT was 3.2 times lower compared to the total number of DEGs obtained in CVO vs. NVO groups.

\section{Molecular functions and pathways predicting developmental capacity}

To unravel relevant molecular functions and pathways in competent in vivo derived embryos, we performed gene ontological enrichment analysis of preferentially expressed genes in $\mathrm{CVO}$ and $\mathrm{NVO}$ groups using the g: Profiler bionformatic tool. Accordingly, those DEGs were found to be mainly involved in ATP production related molecular functions (oxireducatase activity, electron transfer activity, cytochrome c oxidase activity and NADH dehydrogenase activity) (Fig. 5) and KEGG pathways associated with energy metabolism and transformation (glycolysis/glycogenesis, citrate acid cycle, pyruvate metabolism and oxidative phosphorylation), foxo signaling and proteasome) (Fig. 6). Likewise, we have also investigated the relevant molecular functions and pathways in bovine in vitro derived developmentally competent embryos. Gene ontology enrichment analysis showed that preferentially expressed genes in CVT and NVT were found to be involved in translation initiation factor activity, nucleic acid binding, protein binding, actin filament binding and actin filament binding molecular functions (Supplemental Table S3). Moreover, those DEGs were found to be involved in 13 KEGG pathways including protein processing in endoplasmic reticulum, spliceasome, ubiquitone mediated proteolysis and steroid biosynthesis (Fig. 7).

\section{Expression of genes predictive for the developmental capacity in ICM and TE cells}

Mammalian embryos' ability to induce a pregnancy is believed to be dependent on proper specialization the totipotent embryonic cells into pluripotent inner cell mass (ICM) and trophectoderm (TE) cells. This processes is in turn governed by preferential expression of typical molecular signatures in ICM and TE cells [41]. With respect to this, we conducted a meta-analysis by comparing the DEGs identified in embryos of CVO vs. NVO groups with the gene expression outline of ICM and TE cells of in vivo [42] and in vitro [43] derived bovine blastocysts. Interesting, 172 DEGs reported to be differentially expressed between ICM and TE cells of in vivo derived bovine blastocysts [42] and 17 DEGs reported to be by differentially expressed between ICM and TE cells of in vitro derived blastocysts [43] were also found to be differentially expressed between CVO and NVO blastocysts in the present study (Fig. 8a \& b, Supplemental Table S4). Of these, a total of 67 genes including the ribosomal proteins (RPS8, RPS21, RPLP2, RPL39, RPL38, RPL36A, RPL31, RPL30, RPL24, RPL15, RPL13 and $R P L 11$ ) which were upregulated in CVO compared to NVO embryos were also upregulated in ICM compared to TE cells of in vivo derived blastocysts [42]. On the other hand, a total of 82 genes including KRT19, KRT8, CTSZ, KRT18, and MYL6 were downregulated in CVO vs. NVO groups but upregulated in TE vs. ICM cells. Likewise, 14 genes which were downregulated in CVO vs. NVO including ZNF281, NANOG, FOSL1 and $D P Y S$ were upregulated in ICM compared to TE cells whereas 7 genes (RPRD1A, RHOC, PLXDC2, PGRMC1, CYP51A1, C6orf120 and ACLY) which were downregulated in $\mathrm{CVO}$ vs. NVO were also downregulated in ICM vs. TE cells. Functional annotation showed that some of these genes were enriched in distinct pathways namely Foxo signalling pathway, glycolysis/gluconeogenesis, 
Table 2 Differentially expressed gene variants between CVO and NVO groups

\begin{tabular}{llll}
\hline Probe ID & Target ID & Gene & Expression patterns \\
& & symbol & \\
\hline EMBV3_33164 & XM_002697258 & LOC100337465 & $\uparrow$ \\
EMBV3_19607 & NM_001101984 & ALDH3A2 & $\uparrow$ \\
EMBV3_20670 & NM_001075509 & TFAP2C & $\uparrow$ \\
EMBV3_15124 & XM_002694536 & UBE3B & $\uparrow$ \\
EMBV3_11386 & NM_001034296 & NET1 & $\uparrow$ \\
EMBV3_08558 & XM_002692833 & SNX16 & $\uparrow$ \\
EMBV3_30354 & XM_002692428 & SRXN1 & $\uparrow$ \\
EMBV3_29131 & NM_001098069 & CASC3 & $\uparrow$ \\
EMBV3_28415 & NM_174361 & IMPA1 & $\uparrow$ \\
EMBV3_04016 & NM_001101989 & TMEM144 & $\uparrow$ \\
EMBV3_23797 & NM_001102052 & SKP2 & $\downarrow$ \\
EMBV3_07237 & NM_001075133 & PGRMC1 & $\downarrow$ \\
EMBV3_34603 & NM_001110774 & C3Orf57 & $\downarrow$ \\
EMBV3_01743 & NM_001075980 & TM4SF1 & $\downarrow$ \\
EMBV3_41263 & NM_001102503 & LOC520387 & $\downarrow$ \\
EMBV3_33131 & XM_002699713 & MECP2 & $\downarrow$ \\
EMBV3_27274 & NM_001103331 & C6Orf120 & $\downarrow$ \\
EMBV3_21895 & NM_001099036 & NCK2 & $\downarrow$ \\
EMBV3_15031 & NM_001075156 & RPRD1A & $\downarrow$ \\
EMBV3_30252 & XM_002694236 & ZNF281 & $\downarrow$ \\
EMBV3_26127 & NM_001081526 & PCMTD1 & $\downarrow$ \\
EMBV3_14309 & NM_001102147 & FGFR1OP2 & $\downarrow$ \\
EMBV3_02754 & XM_002686473 & THRAP3 & $\downarrow$ \\
EMBV3_25144 & XM_002697779 & LOC100296226 & $\downarrow$ \\
EMBV3_14247 & NM_001083654 & SLC35E3 & $\downarrow$ \\
AN134221 & NM_001098050 & MAML2 & $\downarrow$ \\
EMB11161 & NM_001025319 & CYP51A1 & $\downarrow$ \\
\hline NM_001102223 & CDYL & $\downarrow$ \\
\hline
\end{tabular}

Arrows $\uparrow$ and $\downarrow$ indicate up and down regulation in CVO compared to NVO blastocysts, respectively

beta-Alanine metabolism, pantothenate and CoA biosynthesis, pyrimidine metabolism and fatty acid degradation pathways (Fig. 8c).

The DEGs identified between competent and noncompetent in vitro derived blastocysts (CVT vs. NVT) were also merged to studies of Hosseini et al. [42] and Ozawa et al. [43] . This analysis has shown that a total of 66 and 6 annotated DEGs previously reported to be differentially expressed in ICM vs. TE cells of in vivo [42] and in vitro derived blastocysts [43] were also differentially expressed between CVT and NVT blastocysts in the present study (Fig. 9a \& b, supplemental Table S5). Of those, 28 genes including PPP1CC, ZNF281, $H 3 F 3 B$ and $H 2 A F Z$ reported previously to be enriched in ICM cells [42] were downregulated in CVT compared to NVT embryos whereas 35 genes obtained to be downregulated in CVT vs. NVT embryos in the present study including FERMT2, SLC16A1, SNX4, TXN and PDIA6 were reported to be enriched in TE cells (Supplemental Table S5). Bioinformatic analysis showed that these DEGs were involved in steroid biosynthesis, endocytosis, regulation of actin cytoskeleton, mismatch repair (Fig. 9c).

\section{Molecular signatures reflecting environmental conditions in competent bovine embryos}

'Understanding the differences in the gene expression outline between developmentally competent in vitro and in vivo originated blastocyst is suggested to be useful to identify gene expression signatures associated with pathophysiological postnatal consequences caused by the environment during in vitro embryo production. Therefore, here we investigated differences in terms of gene expression signatures specifically affected by developmental environment in competent in vitro derived blastocysts (CVT) vs. competent vivo derived ones (CVO). Including novel transcripts (NTRs), alternative 3'-UTR events, Indel-type splice variants and pseudogenes, a total of 1066 probes associated with 937 transcripts were differentially expressed between competent in vivo and in vitro derived embryo groups indicating differences although both groups had resulted in establishment of pregnancy. The expression trend of $83.2 \%$ of all DEGs including RPS27A, RPS21, RPS13, EEF1A1 and CYCS was reduced whereas the expression of $16.7 \%$ of all DEGs including SEMA6C, TPRF, NFATC4 and SMARCA2 was increased in CVT vs. CVO samples (Supplemental Fig. 1, Supplemental Table S6). In addition, a total of 50 DEGs including RPLPO, COX5A, ATP5J2 and ATP5C1 represented indel type splice variants (Table 7) whereas a total of 83 differentially expressed probes including those associated with TPM4, SLC31A1, INA, CS, TP53INP1, NCOA1, ATF1 and SLC1A3 genes represented alternative 3 '-UTR variants (Supplemental Table S7).

\section{Expression of gene cluster reflecting the environmental conditions in competent bovine embryos}

In addition to characterize individual differential expressed genes as novel transcripts (NTR), alternative 3 '-UTR events, indel type splice variants or pseudogenes, we have also identified some gene cluster bearing similar expression patterns in one sample group relative to another one which is a step forward for selecting promising candidate genes associated with the trait of interest. Accordingly, we have investigated the expression patterns of genes which share similar characteristics and biochemical functions. Thus, a detailed analysis has shown that several cluster of genes including ribosomal proteins $(n=36)$, zinc fingers $(n=9)$, solute carriers $(n=$ 
Table 3 Differentially expressed 3'-UTR alternative variants between CVO and NVO groups

\begin{tabular}{|c|c|c|c|}
\hline Probe ID & Target ID & $\begin{array}{l}\text { Gene } \\
\text { symbol }\end{array}$ & Expression patterns \\
\hline EMBV3_15895 & NM_001076288:853^956 & ZBTB8OS & $\uparrow$ \\
\hline EMBV3_11755 & NM_001103313:118^183 & STAU2 & $\downarrow$ \\
\hline EMBV3_29618 & XM_002690797:448^620 & RTF1 & $\uparrow$ \\
\hline EMBV3_23690 & NM_001040581:162^233 & RPS 21 & $\uparrow$ \\
\hline EMBV3_04521 & NM_001034434:91^236 & RPL30 & $\uparrow$ \\
\hline EMBV3_11916 & NM_001046138:573^772 & RHOC & $\downarrow$ \\
\hline EMBV3_04120 & NM_001034266:602^718 & PSMB4 & $\uparrow$ \\
\hline EMBV3_38466 & NM_174749:398^529 & PRDX5 & $\uparrow$ \\
\hline EMBV3_37565 & NM_001025325:256^495 & PLAC8 & $\uparrow$ \\
\hline EMBV3_30094 & NM_001025325:256^380 & PLAC8 & $\uparrow$ \\
\hline EMBV3_14878 & NM_001034440:600^645 & PEBP4 & $\downarrow$ \\
\hline EMBV3_12712 & NM_001034384:651^728 & NOL7 & $\uparrow$ \\
\hline EMBV3_00366 & NM_001038133:297^732 & NIT2 & $\downarrow$ \\
\hline EMBV3_13552 & XM_002686892:558^606 & MYL7 & $\uparrow$ \\
\hline EMBV3_35400 & XM_002686892:374^478 & MYL7 & $\uparrow$ \\
\hline EMBV3_16450 & NM_175780:406^572 & MYLG & $\uparrow$ \\
\hline EMBV3_15619 & NM_175780:458^502 & MYL6 & $\uparrow$ \\
\hline EMBV3_22522 & NM_175780:413^569 & MYL6 & $\uparrow$ \\
\hline EMBV3_27866 & NM_175780:273^550 & MYL6 & $\uparrow$ \\
\hline EMBV3_27828 & NM_175780:392^627 & MYL6 & $\uparrow$ \\
\hline EMBV3_40962 & NM_175780:313^568 & MYL6 & $\uparrow$ \\
\hline EMBV3_35847 & NM_001076018:227^306 & MTHFD1L & $\uparrow$ \\
\hline EMBV3_27125 & NM_001046508:169^252 & MRPS18C & $\uparrow$ \\
\hline EMBV3_07488 & NM_001075276:429^789 & MRPL55 & $\uparrow$ \\
\hline EMBV3_08656 & XM_002692789:248^375 & MGC148714 & $\uparrow$ \\
\hline EMBV3_08932 & XM_002685429:193^371 & LOC781039 & $\downarrow$ \\
\hline EMBV3_04189 & XM_002694113:286^391 & LOC616065 & $\uparrow$ \\
\hline EMBV3_35024 & XM_002685423:386^787 & LOC100337018 & $\downarrow$ \\
\hline EMBV3_26700 & XM_002685421:278^542 & LOC100336997 & $\downarrow$ \\
\hline EMBV3_32203 & XM_002690260:82^219 & ILZORA & $\downarrow$ \\
\hline EMBV3_13795 & NM_001101264:282^515 & FERMT2 & $\uparrow$ \\
\hline EMBV3_33655 & NM_174217:747^992 & $E Z R$ & $\uparrow$ \\
\hline EMBV3_19809 & NM_001075795:717^795 & EIF4E2 & $\uparrow$ \\
\hline EMBV3_41916 & NM_001015586:55^362 & DSTN & $\uparrow$ \\
\hline EMBV3_36018 & NM_001033763:224^944 & DNAJB1 & $\downarrow$ \\
\hline EMBV3_42215 & NM_175807:98^187 & COX7A2 & $\uparrow$ \\
\hline EMBV3_03749 & NM_001077831:264^339 & COX6A1 & $\uparrow$ \\
\hline EMBV3_34344 & NM_001002891:521^562 & COX5A & $\uparrow$ \\
\hline EMBV3_08339 & NM_001002891:400^444 & COX5A & $\uparrow$ \\
\hline EMBV3_28626 & NM_001078036:814^858 & $C O B L$ & $\uparrow$ \\
\hline EMBV3_29989 & NM_001001855:292^409 & BIRC5 & $\uparrow$ \\
\hline EMBV3_28386 & NM_001113719:163^210 & ATP5J2 & $\uparrow$ \\
\hline EMBV3_25301 & NM_174724:350^412 & ATP5H & $\uparrow$ \\
\hline EMBV3_07361 & NM_176649:158^336 & ATP5G1 & $\uparrow$ \\
\hline
\end{tabular}

Arrows $\uparrow$ and $\downarrow$ indicate up and down regulation in CVO compared to NVO blastocysts, respectively 


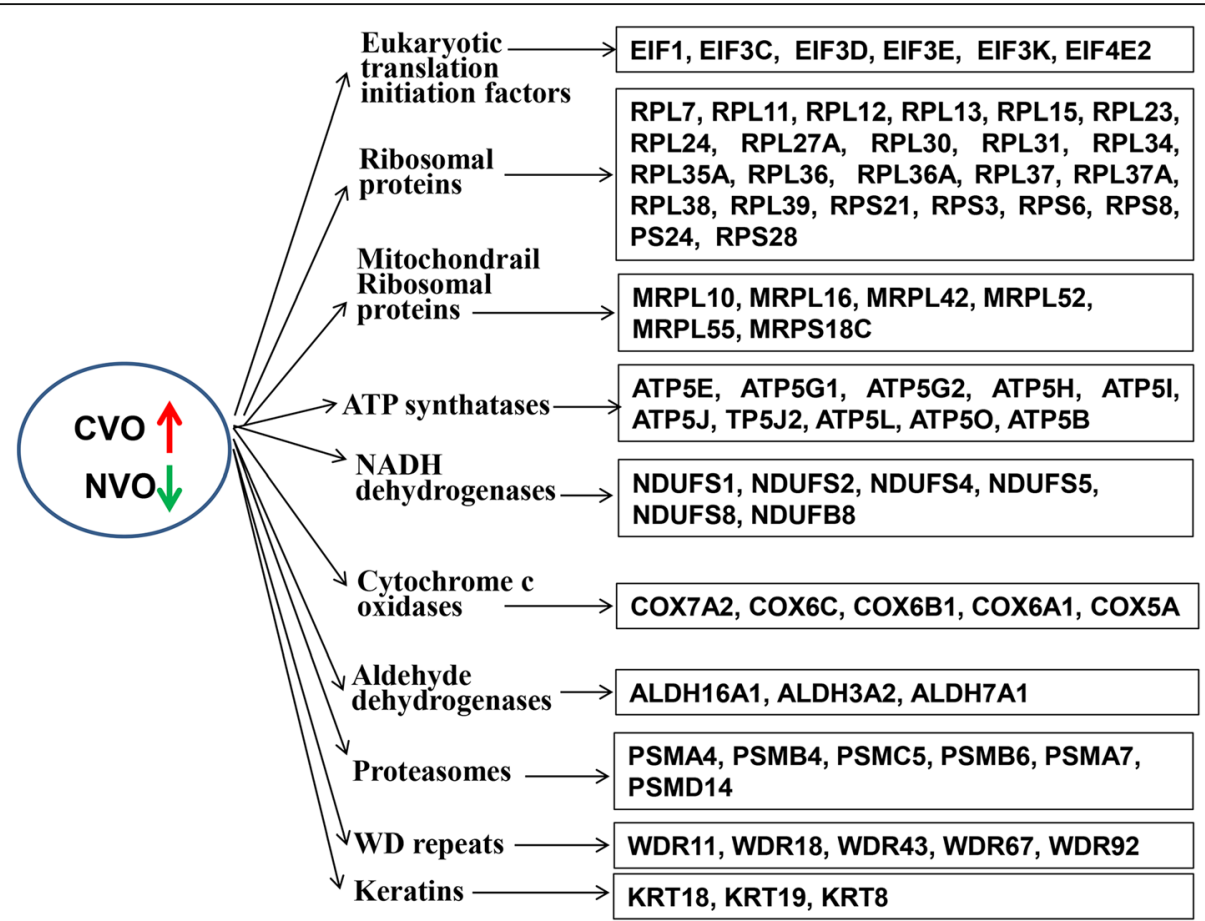

Fig. 3 Gene clusters significantly enriched in in vivo derived competent embryos (CVO) compared to none competent (NVO) ones

7), mitochondrial ribosomal proteins $(n=9)$, eukaryotic translation initiation factor $(n=5)$, nuclear ribonucleoprotein $(n=6)$ and NADH dehydrogenase $(n=4)$ were reduced in the CVT group compared to the CVO ones (Fig. 10). Of the 36 differentially expressed ribosomal proteins, the expression of ribosomal proteins including RPS27A, RPS21, RPS13, RPL12, RPL27, RPL7, RPS24, RPS3, RPS29 and RPS 21 exhibited 4-8 fold change reduction in CVT.

\section{Molecular functions and pathways reflecting} environmental conditions of competent bovine embryos Gene ontology enrichment analysis showed that those differentially expressed genes between CVT and CVO samples were found to be involved in biological processes associated with metabolism, ATP production, cell cycle related activities, and protein synthesis (Fig. 11a, supplemental Table S8). In addition, those DEGs were also found to be involved in molecular functions including binding activity, oxireductase activity, cytocrom-c reductase activity, electron transfer activity and catalytic activities (Fig. 11b, supplemental Table S9). In line with that, these DEGs were involved in distinct molecular pathways including translation, energy metabolism, transport and catabolism, cell growth and death, folding, sorting and degradation, carbohydrate metabolism, replication, and repair (Supplemental Fig. 2).

Molecular signatures reflecting environmental conditions exclusively in competent bovine embryos

Identification of genes being exclusively differentially expressed between competent in vivo derived and competent in vitro derived embryos (CVT vs. CVO) and between non-competent in vivo derived embryos and noncompetent in vitro derived embryos (NVT vs. NVO) was done to identify genes which were specifically affected by the culture environment without being conflictive with further development if aberrantly expressed. The result from

Table 4 Preferentially expressed probes in CVT and NVT groups

\begin{tabular}{llll}
\hline & Enriched in CVT & Enriched in NVT & Total \\
\hline Constitutive (not discriminating variants) & 15 & 157 & 172 \\
Novel gene; evidence: embryonic ESTs (NTR) & 3 & 28 & 31 \\
Alternative 3'UTR events (genes) & 1 & 18 & 19 \\
Indel type splice variants & 4 & 0 & 4 \\
Total differentially expressed probes & 23 & 203 & 226 \\
\hline
\end{tabular}




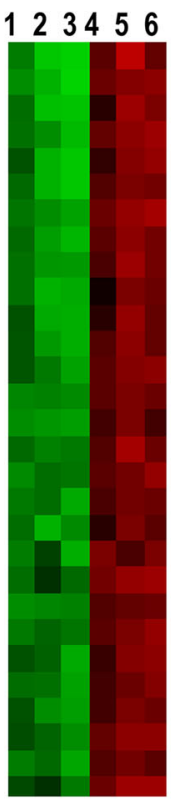

Gene symbol
TPT1
PDIA6
HSP90AA1
CALM
GATA-6
LOC100297344
LOC100297240
EIF4A1
CTNNA1
SLC2A3
RPL26
HNRNPA3
MAD2
TMSB4X
GNG5
TPT1
DSG2
VCL
CBX3
TMEM49
ENPEP
ITM2B
CAPZA1
CSRP2
SPETin15
LOC784704
PICALM
TMSB4X
H3F3B

$\begin{array}{rr}\log _{2} \text { FC } & \text { P. Value } \\ -2.23 & 0.001 \\ -2.09 & 0.000 \\ -1.83 & 0.001 \\ -1.72 & 0.000 \\ -1.71 & 0.002 \\ -1.70 & 0.002 \\ -1.68 & 0.000 \\ -1.58 & 0.000 \\ -1.43 & 0.000 \\ -1.42 & 0.002 \\ -1.41 & 0.002 \\ -1.39 & 0.001 \\ -1.30 & 0.000 \\ -1.30 & 0.000 \\ -1.28 & 0.001 \\ -1.27 & 0.000 \\ -1.26 & 0.000 \\ -1.26 & 0.000 \\ -1.23 & 0.002 \\ -1.23 & 0.001 \\ -1.21 & 0.000 \\ -1.20 & 0.000 \\ -1.20 & 0.000 \\ -1.20 & 0.002 \\ -1.19 & 0.000 \\ -1.19 & 0.000 \\ -1.19 & 0.000 \\ -1.19 & 0.000 \\ -1.17 & 0.001\end{array}$

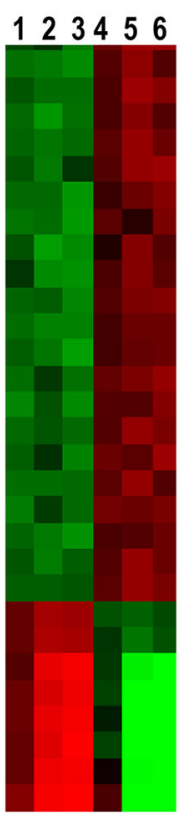

Gene symbol
PPP2CA
NPM1
LOC100299038
PCNA
MAD2L1
ANXA5
SAR1B
LOC782479
HNRPDL
LOC618550
LOC100297368
LOC785421
ZBTB10
SEPTIN7
PCNA
CTGF
EIF3M
SEPP1
ARF1
NONO
PSMA2
TRIP6
HSD11B1L
EGLN1
LOC785926
EIF1AD
OTUB1
LOC100294941
STAT

\begin{tabular}{cl} 
Log $_{2} \mathrm{FC}$ & P. Value \\
-1.13 & 0.000 \\
-1.12 & 0.000 \\
-1.11 & 0.000 \\
-1.10 & 0.000 \\
-1.09 & 0.001 \\
-1.09 & 0.000 \\
-1.08 & 0.001 \\
-1.08 & 0.002 \\
-1.08 & 0.001 \\
-1.07 & 0.000 \\
-1.06 & 0.000 \\
-1.06 & 0.001 \\
-1.06 & 0.000 \\
-1.05 & 0.000 \\
-1.03 & 0.000 \\
-1.03 & 0.001 \\
-1.02 & 0.000 \\
-1.02 & 0.000 \\
-1.01 & 0.000 \\
-1.01 & 0.000 \\
-1.01 & 0.000 \\
1.15 & 0.002 \\
1.27 & 0.002 \\
4.32 & 0.002 \\
4.55 & 0.002 \\
4.68 & 0.002 \\
4.71 & 0.002 \\
4.74 & 0.002 \\
5.15 & 0.002 \\
& \\
\hline
\end{tabular}

The colors scale:

Min $=8.34$

Fig. 4 Molecular signature associated with developmental capacity of in vitro derived embryos. The heatmap indicates the expression patterns of the top 58 differentially expressed genes between CVT and NVT. Numbers 1, 2, 3 indicate three biological replicates hybridization whereas $1 \mu \mathrm{g}$ of labelled Cy-5 labelled CVT samples were hybridized with $1 \mu \mathrm{g}$ of Cy-3 labelled NVT samples. Numbers 4, 5, 6 indicate dye-swaps in which $1 \mu \mathrm{g}$ of labelled Cy-3 labelled CVT samples were hybridized with $1 \mu \mathrm{g}$ of Cy-5 labelled NVT samples

this analysis indicated as much as four times more exclusively differentially expressed annotated genes between CVT vs. CVO compared to those detected when comparing NVT vs. NVO groups (Fig. 12a). Exclusively differentially expressed genes in CVT vs. CVO include several gene clusters such as ribosomal proteins, zinc fingers, solute carriers, mitochondrial ribosomal proteins, eukaryotic translation initiation factors, nuclear ribonucleoprotein and NADH dehydrogenase. In agreement, genes exclusively differentially expressed in the CVT vs. CVO groups were found to be involved in several pathways including oxidative phosphorylation, ubiquitin mediated proteolysis, cellular senescence, and proteasome pathways (Fig. 12b).

\section{Molecular signatures reflecting environmental conditions in non-competent embryos}

The transcriptome profile comparison between noncompetent in vitro (NVT) and non-competent in vivo blastocysts identified a total of 495 differential expressed probes encompassing 307 annotated and 188 novel transcripts (NTRs). Of these, $85.8 \%$ were down regulated in NVT group compared to NVO ones. Since, both NVT and NVO were confirmed not to end up in an initial pregnancy, different expressed genes between these two blastocysts groups is purely due to contrasting culture conditions. Among those, a total of 158 differentially expressed annotated genes were found to be common in the CVT vs. CVO comparison and. These genes were found to be preferentially involved in 6 KEGG pathways including citrate cycle (TCA cycle), carbon metabolism and apoptosis pathways (Fig. 12c).

\section{Expression of genes reflective for the developmental environment in ICM and TE}

We also superimposed the genes preferentially expressed between the competent vivo and in vitro blastocysts

Table 5 Differentially expressed gene variants between CVT and NVT groups

\begin{tabular}{llll}
\hline Probe ID & Target ID & Gene symbol & Expression patterns \\
\hline EMBV3_09669 & NM_001034719:575^658 & OTUB1 & $\uparrow$ \\
EMBV3_11755 & NM_001103313:118^183 & STAU2 & $\uparrow$ \\
EMBV3_12635 & NM_001035469:214^341 & TRIP6 & $\uparrow$ \\
EMBV3_19031 & NM_206969:327^438 & HSD11B1L & $\uparrow$ \\
\hline
\end{tabular}

Arrow $(\uparrow)$ indicates upregulation genes in the CVT group compared to the NVT ones 
Table 6 Differentially expressed 3'-UTR alternative variants between CVT and NVT groups

\begin{tabular}{llll}
\hline Probe ID & Gene symbol & Description & Expression patterns \\
\hline EMBV3_30252 & ZNF281 & Zinc finger protein 281 & $\downarrow$ \\
EMBV3_40010 & TMEM170A & TMEM170 transmembrane protein 170A & $\downarrow$ \\
EMBV3_03965 & SYPL1 & Synaptophysin-like 1 & $\downarrow$ \\
EMBV3_08091 & SYAP1 & Synapse associated protein 1, SAP47 homolog (Drosophila) & $\downarrow$ \\
EMBV3_42313 & SNX4 & Sorting nexin 4 & $\downarrow$ \\
EMBV3_14247 & SLC35E3 & Solute carrier family 35, member E3 & $\downarrow$ \\
EMBV3_05094 & SEPTIN7 & Septin 7 & $\downarrow$ \\
EMBV3_13169 & RELL1 & RELT-like 1 & $\downarrow$ \\
EMBV3_21053 & PPP1CC & Protein phosphatase 1, catalytic subunit, gamma isoform & $\downarrow$ \\
EMBV3_25376 & KCTD8 & Potassium channel tetramerisation domain containing 8 & $\downarrow$ \\
EMBV3_30092 & PLEKHF2 & Pleckstrin homology domain containing, family F (with FYVE domain) member 2 & $\downarrow$ \\
EMBV3_33562 & PHF3 & PHD finger protein 3 & $\downarrow$ \\
EMBV3_16278 & NCOA4 & Nuclear receptor coactivator 4 & $\downarrow$ \\
EMBV3_07677 & M6PR & CDMPR MGC140730 mannose-6-phosphate receptor (cation dependent) & $\downarrow$ \\
EMBV3_41263 & LOC520387 & KIAA0528-like & $\downarrow$ \\
EMBV3_26821 & DSG2 & Desmoglein 2 & $\downarrow$ \\
EMBV3_11161 & CYP51A1 & Cytochrome P450, family 51, subfamily A, polypeptide 1 & $\downarrow$ \\
EMBV3_35542 & CDYL & Chromodomain protein, Y-like & $\downarrow$ \\
EMBV3_16966 & CANX & Calnexin & $\downarrow$ \\
\hline A & & $\downarrow$ \\
\end{tabular}

Arrow $(\downarrow)$ indicates the downregulation genes in CVT compared to NVT blastocysts

(CVT vs. CVO) to those genes previously reported to be differentially expressed for ICM vs. TE cell of in vivo [42] and in vitro derived bovine blastocysts [43], respectively as indicated above. Accordingly, a total of 248 and 14 DEGs detected in CVT vs. CVO were identified as differentially expressed between ICM and TE cells of in vivo [42] and in vitro [43] derived bovine blastocysts, respectively (Fig. 13a \& b). Of these, 157 genes have been shown to be enriched in ICM cells, whereas 91 DEGs have been reported to be enriched in TE cells of

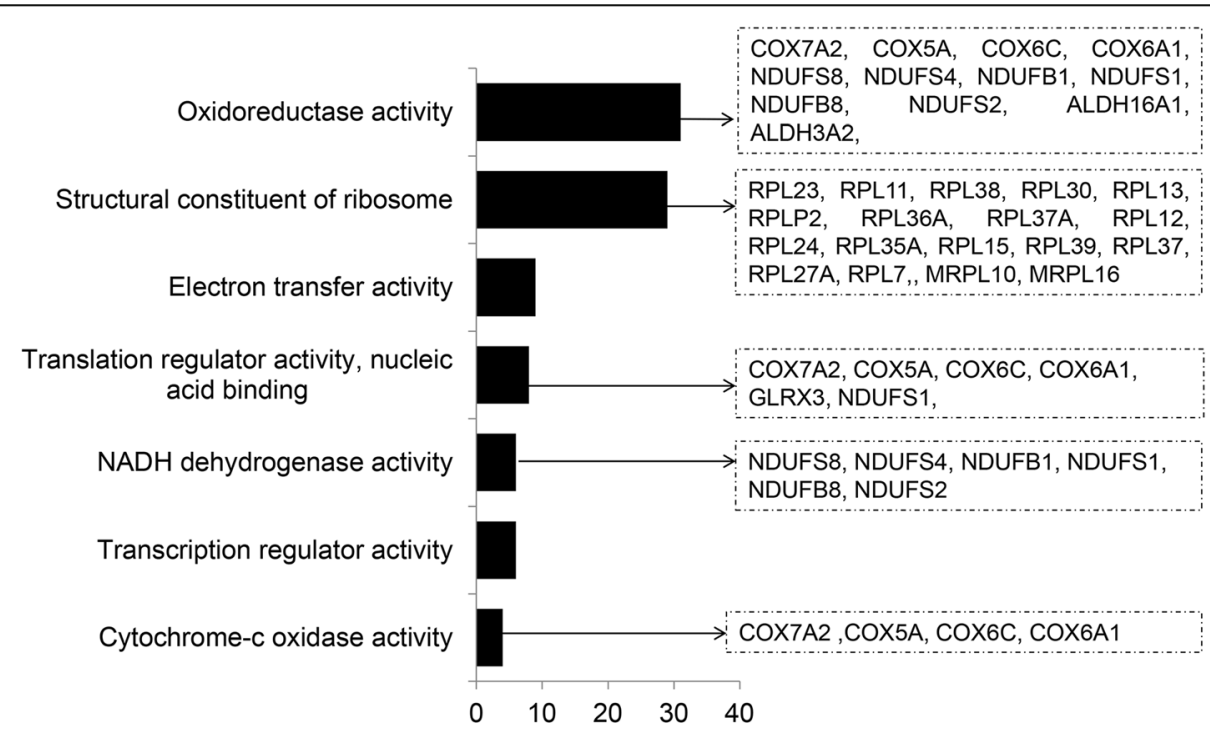

Number of differentially expressed genes

Fig. 5 Molecular functions significantly enriched by differentially expressed genes in competent in vivo derived embryos (CVO vs. NVO). Lists of genes on the right indicate differentially expressed genes involved within these distinct molecular functions 


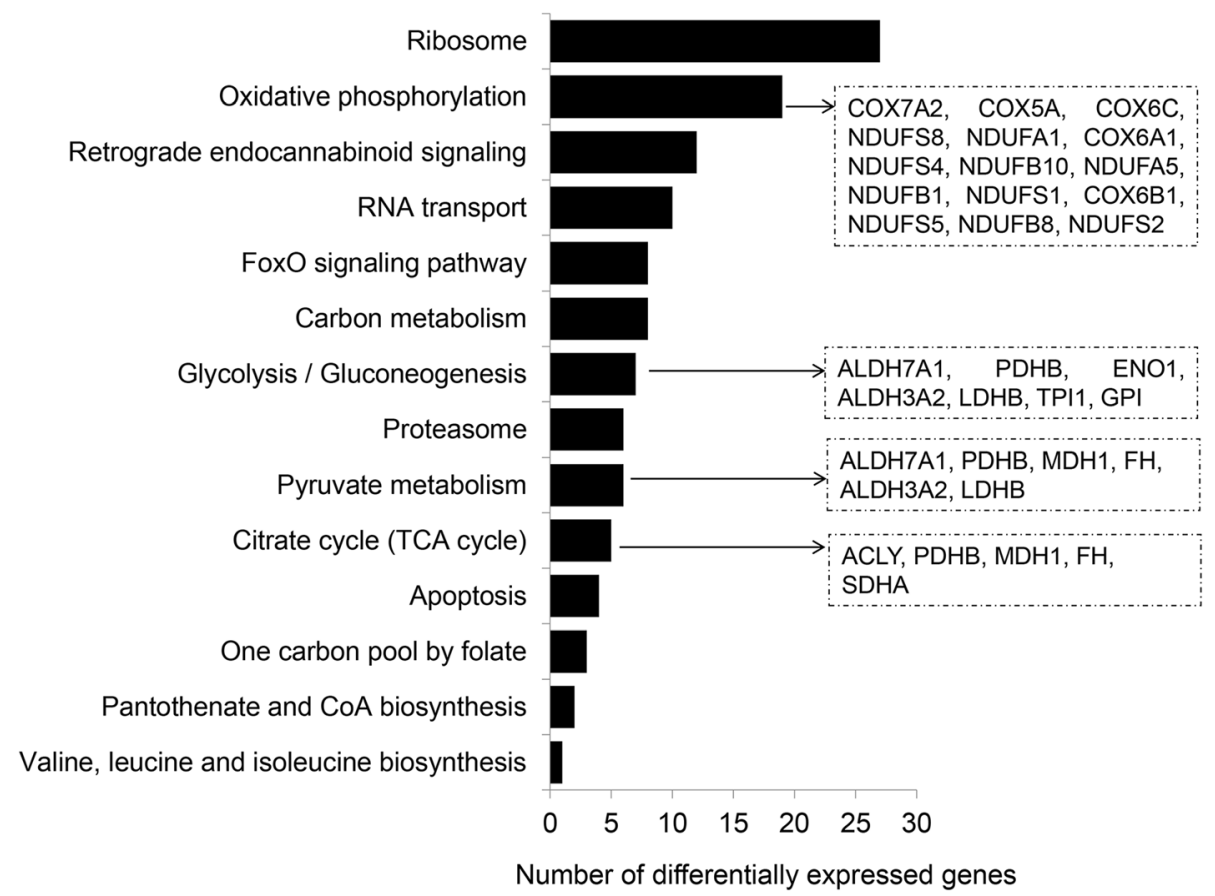

Fig. 6 Molecular pathways significantly enriched by differentially expressed genes in competent in vivo derived embryos (CVO vs. NVO). Lists of genes on the right indicate differentially expressed genes involved within these distinct molecular pathways

in vivo derived blastocysts, respectively (Fig. 13a, Supplemental Table S10). These genes are enriched in ribosome, cysteine, and methionine metabolism, one carbon pool by folate focal adhesion and oxidative phosphorylation pathways (Fig. 13c).

\section{Discussion}

Mammalian preimplantation embryogenesis involves entire developmental stages from the formation of the diploid zygote up to formation of the blastocyst. After the blastocysts stage, the trophectoderm of the developing embryo

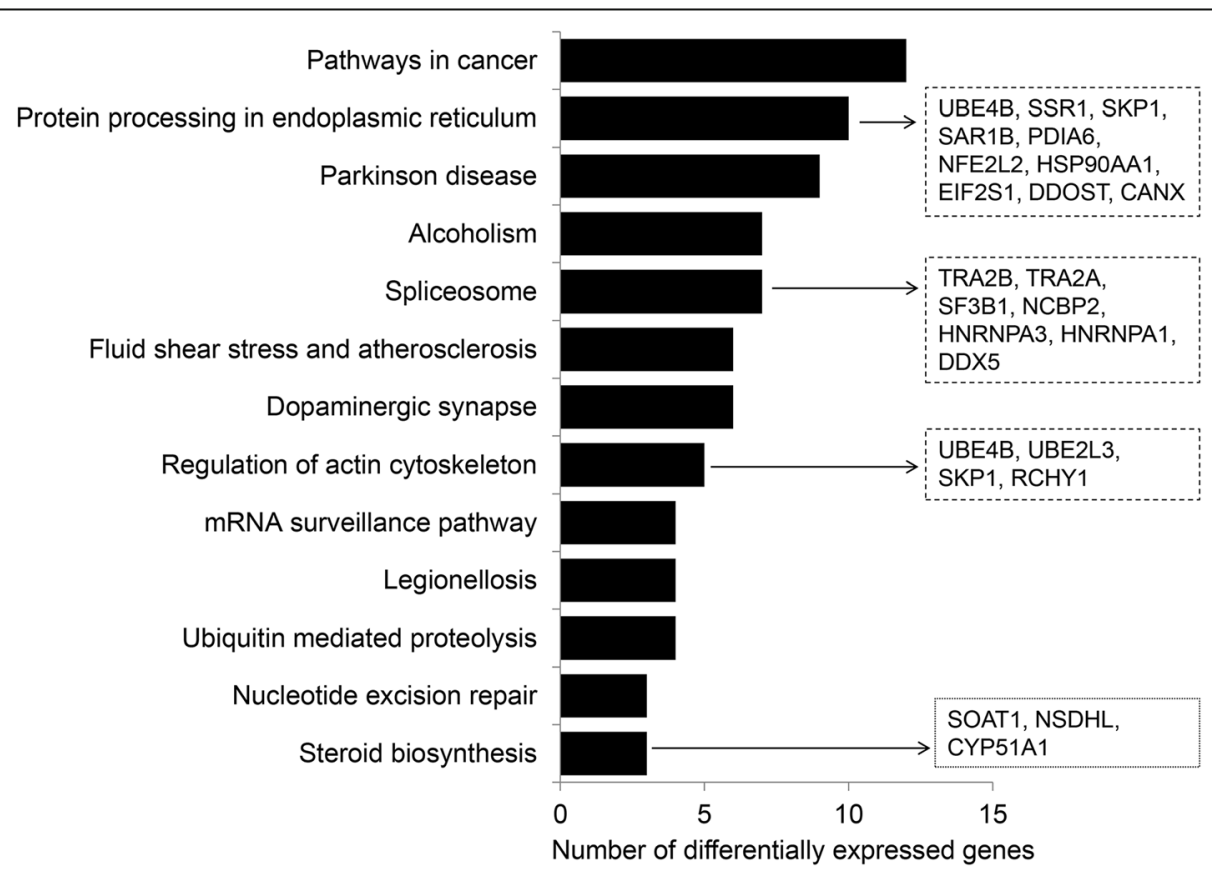

Fig. 7 Molecular functions significantly enriched by differentially expressed genes specifically in competent in vitro derived embryos (CVT vs. NVT). Lists of genes on the right indicate differentially expressed genes involved within these distinct molecular functions 


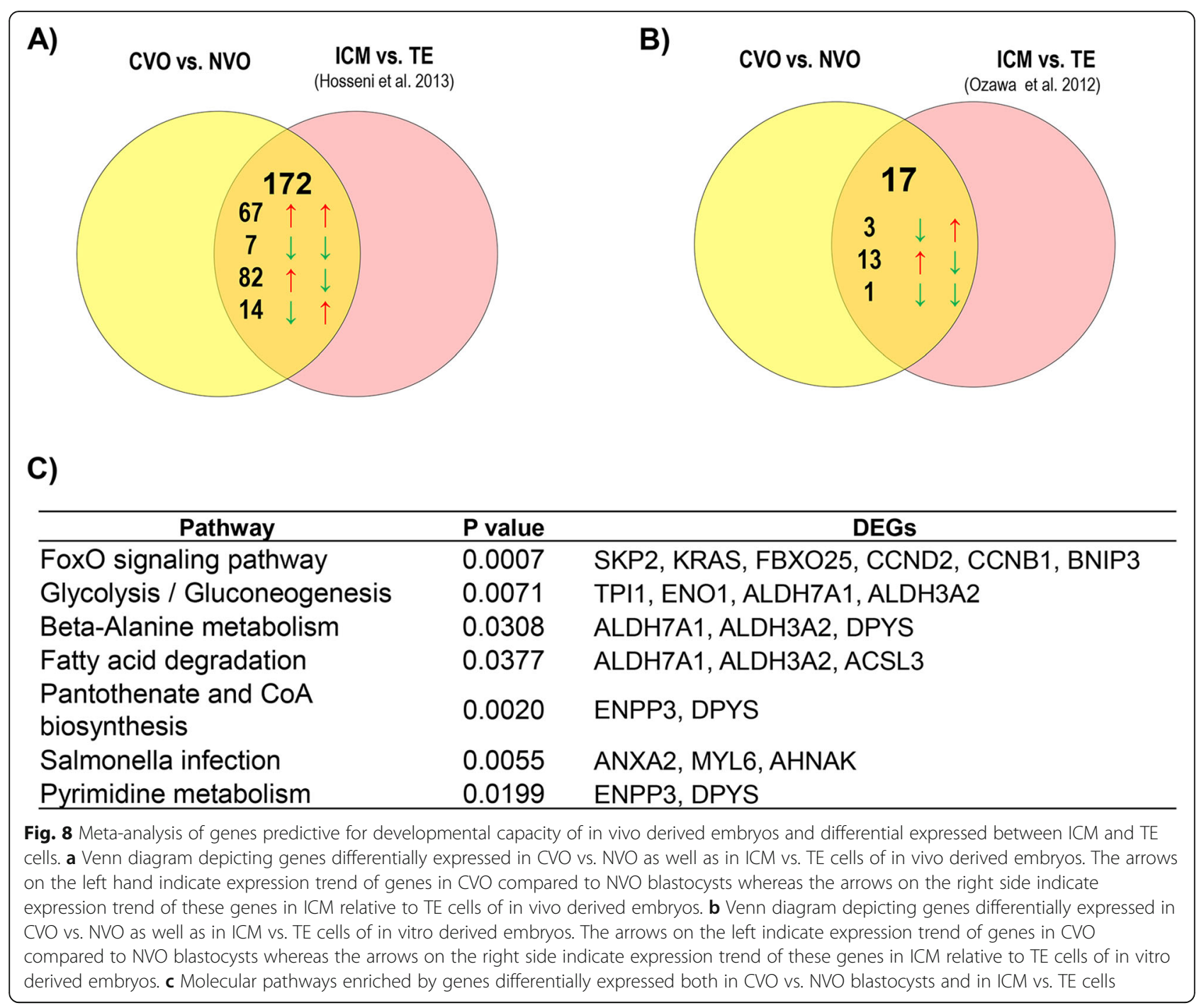

gives rise to extra embryonic tissues and the epiblast differentiates into somatic lineages and the germline [44]. It is known that during the first successive cleavage stages, the embryo depends on the maternal stores accumulated during oocyte growth. After activation of its own genome, the embryo depends on transcripts and proteins that are de novo synthesized by the embryonic cells which begins at 48 cell stage in humans [45, 46], at 2-cell in mouse [47] and $8-16$ cell stages in bovine [48]. For instance, a progressive increase in gene expression takes place during the early cleavage stages and morulae formation of mouse embryos [49]. This suggests that spatial and temporal dynamic expression of developmentally related genes and successful transitions from maternal to embryonic transcription is critical for successful embryonic development. Thus, identification of those transcripts that could favour embryonic development and sustain pregnancy could be an important step for developing genetic markers for selecting developmentally competent embryos for increasing pregnancy rates after embryo transfer. Not only the intrinsic developmental capacity, but also the developmental environment has been reported to modulate an embryo's gene expression outline. Thus, interpretation of an embryo's transcriptome in relation to its developmental capacity, while neglecting environmental conditions might lead to a wrong conclusion. Similarly, interpretation of embryos transcriptome profiles modulated by environmental conditions, while neglecting an embryo's intrinsic developmental capacity might also lead to false results. Therefore, here we aimed to unravel specific molecular signatures of the bovine embryo and their predictive power with respect to its developmental capacity, contrasting environments and their interaction.

\section{Molecular signatures reflecting embryos' developmental environment}

Although in vitro produced embryos have some potential to develop to term, numerous reports elsewhere have reported prenatal and postnatal consequences in pregnancies 


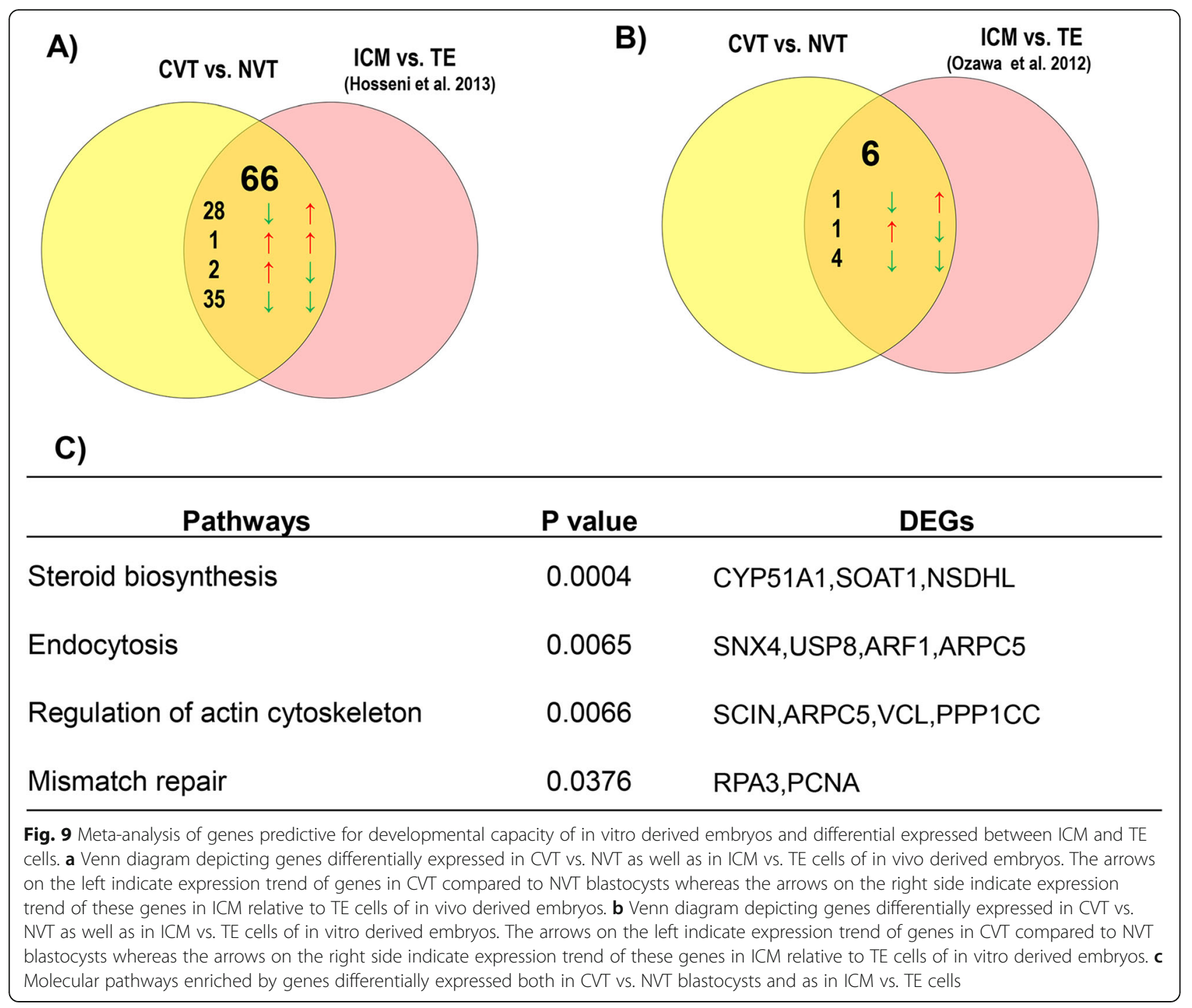

established after transfer of in vitro derived embryos. Indeed, negative effects of the none-physiological in vitro culture environment on an embryo's developmental potential could be a consequence of alterations of the gene expression outline or because of epigenetic modifications. In this respect, several candidate genes and large scale transcriptome profile analysis approaches [18-24] and DNA methylation studies [25-30] have proved profound effects of culture conditions on gene expression patterns and epigenetic profiles in the resultant blastocysts. However, in all these studies, no relevance was given to the developmental capacity of the individual embryo taken for (epi-)genome expression analysis. It is generally accepted, that lower proportions of in vitro derived embryos end up in calf delivery after transfer to recipients (about 15-41\%) compared to in vivo derived counterparts (about 46\%), as reviewed

Table 7 Differentially expressed probes between CVT and CVO groups

\begin{tabular}{llll}
\hline & Enriched in CVT & Enriched in CVO & Total \\
\hline Constitutive (not discriminating variants) & 124 & 562 & 686 \\
Novel gene; evidence: embryonic ESTs (NTR) & 54 & 193 & 247 \\
Alternative 3'UTR events (genes) & 15 & 68 & 83 \\
Indel type splice variants & 18 & 32 & 50 \\
Pseudogenes & 0 & 28 & 28 \\
Total differentially expressed probes & 211 & 855 & 1066 \\
\hline
\end{tabular}




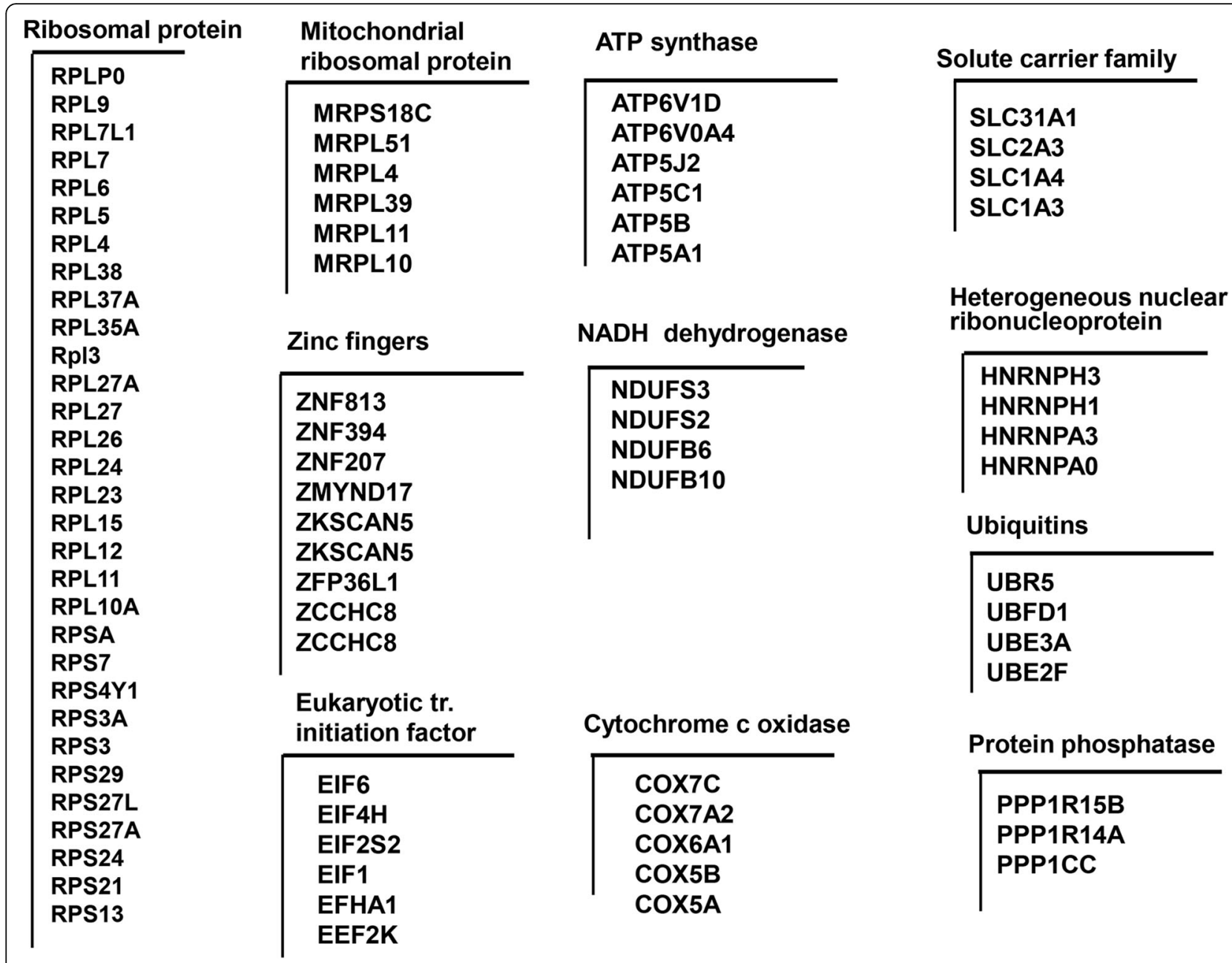

Fig. 10 Arrays of gene cluster reflecting environmental conditions in competent embryos (CVT vs. CVO)

elsewhere [50]. Thus, to avoid incorrectly interpreting an embryo's environmental condition-induced transcriptome profile changes, it is necessary to consider only embryos of comparable developmental capacity for comparative gene expression analysis. On the contrary, comparative gene expression profiles and epigenetic studies in the in vitro and in vivo originated embryos have usually been performed without determining the individual developmental competence of the embryos. Consequently, the results derived from these studies are a mixture of both competent and non-competent embryos. Conversely, in the present study, we investigated the transcriptome profile differences between competent in vivo (CVT) and in vitro $(\mathrm{CVO})$ derived embryos as well as non-competent in vivo (NVT) and in vitro (NVO) derived embryos separately. Interestingly, the total number of differentially expressed genes in those comparisons revealed that an embryo's transcriptome profile specifically modulated by the developmental environment seems to be more divergent in competent embryos than in non-competent ones (Fig. 12). In addition, several clusters of genes including those involved in ribosomal protein synthesis, ATP generating activities, and those involved in oxidative phosphorylation (Fig. 10) and several gene variants (Supplemental Table S11) were exclusively downregulated in blastocysts cultured in vitro. However, the main question that could be raised with respect to this issue is, why competent in vitro derived blastocysts exhibited remarkable gene expression differences compared to competent in vivo ones, yet both resulted in pregnancy establishment. This expression difference between competent in vitro and in vivo derived embryos may indicate a possible cause for postnatal abnormalities sometimes observed in pregnancies established after transfer of in vitro derived embryos. Although, both competent in vivo and in vitro blastocysts are capable of inducing robust pregnancies, reports have shown that some abnormal outcomes after transfer of in vitro derived embryos, including postnatal consequences, prolonged gestations, fetal overgrowth such as the large offspring syndrome phenomena in cattle, longer hind legs in horses, metabolic disturbances including 
A)

Phosphorus metabolic process

RNA biosynthetic process

Phosphorylation

Proteolysis

Oxidation-reduction process

Cellular protein localization Cell cycle

Chromosome organization Cell morphogenesis

Ribosome biogenesis

Embryo development

Mitochondrion organization

Cellular respiration

Cytoplasmic translation

Ribosome assembly

Tricarboxylic acid cycle

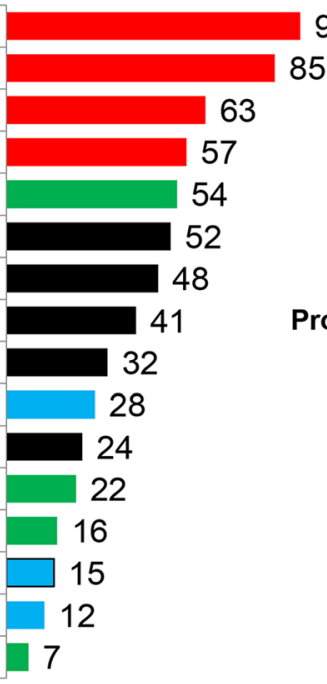

93

85

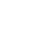

(n)

Nucleotide binding

Carbohydrate derivative binding

Structural molecule activity

Oxidoreductase activity

Protein-containing complex binding

Coenzyme binding

Microtubule binding

Isomerase activity

Translation regulator activity

Electron transfer activity 11

NAD binding 9

Cytochrome-c oxidase activity 7

DNA N-glycosylase activity | 4

Fig. 11 Biological processes (a) and molecular functions (b) significantly enriched by differentially expressed genes specifically modulated by the environmental conditions in competent embryos (CVT vs. CVO). Red, green, black and blue bars in Fig. A indicate functions associated with metabolism, energy production, cell cycle related activities and protein synthesis, respectively whereas yellow and black bars in Fig. B indicate binding and enzymatic activities respectively

A)

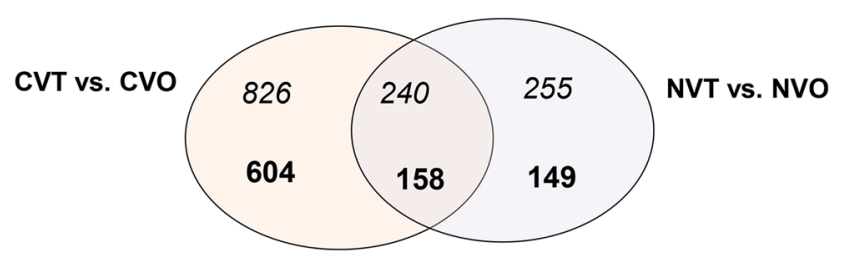

B)

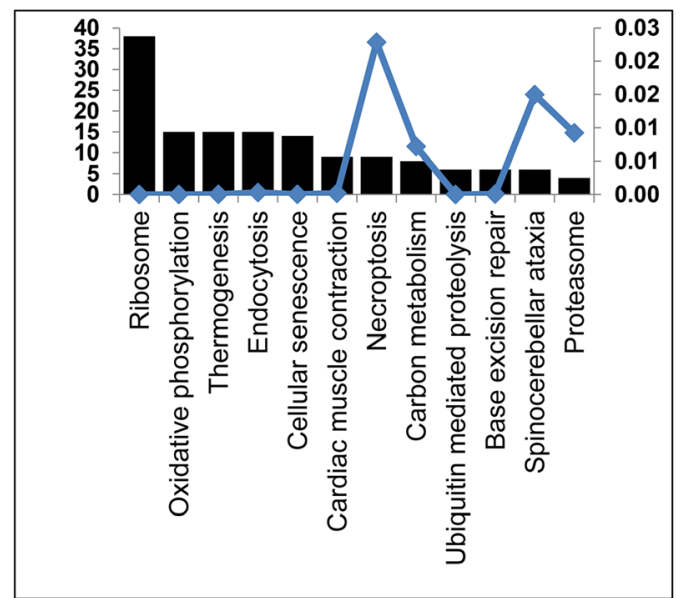

C)

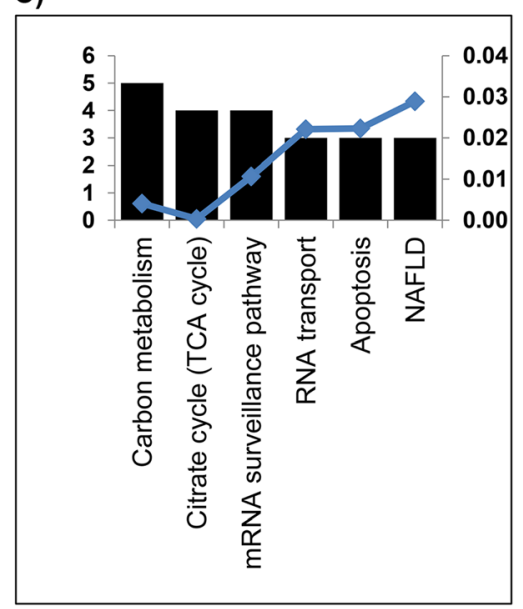

Fig. 12 Summary of (a) differential expressed genes modulated by environmental conditions in competent embryos (left, CVT vs. CVO) and noncompetent embryos (right, NVT vs. NVO). Venn diagram reports also number of genes differential expressed in common (center). Total numbers of differential expressed probes for each section are presented in italic whereas annotated ones are indicated in bold. b Molecular pathways significantly enriched by genes affected by environmental conditions exclusively in competent embryos (CVT vs. CVO). c Molecular pathways significantly enriched by genes affected by environmental conditions both in competent and non-competent embryos. Black bars indicate numbers of differentially expressed genes and blue dots indicate adjusted $p$ values. NAFLD: Non-alcoholic fatty liver disease 

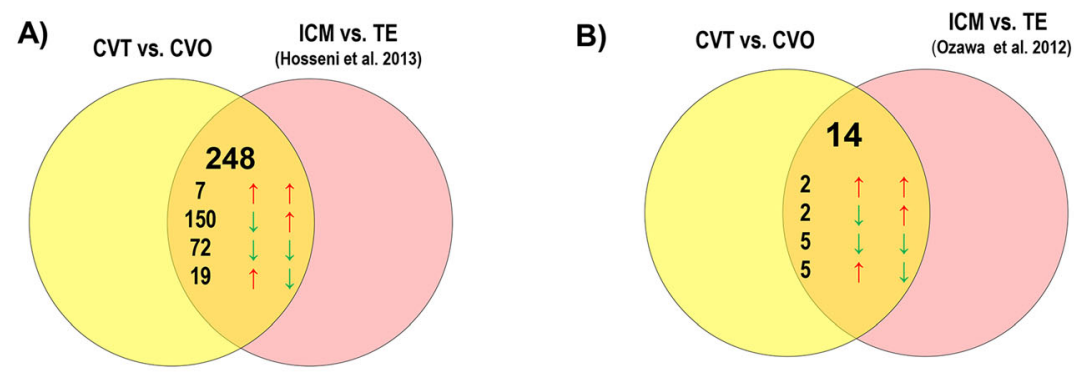

\begin{tabular}{lll}
\hline \multicolumn{1}{c}{ Pathways } & P value & \multicolumn{1}{c}{ DEGs } \\
\hline Ribosome & 0.0000 & RPL11, RPL13A, RPL15, RPL24, RPL38, RPL6, \\
Cysteine and methionine metabolism & 0.0024 & AHCY, AHCPL, RPS21, RPS27L, RPS7, RPSA \\
Fluid shear stress and atherosclerosis & 0.0025 & CALM1, CAV1, CTNNB1, CTSL2 \\
One carbon pool by folate & 0.0027 & ALDH1L2, MTFMT, MTHFD1L \\
Collecting duct acid secretion & 0.0051 & ATP6VOA4, ATP6V1B2 \\
Carbon metabolism & 0.0099 & GCSH, GLDC, PDHA1, PGAM1, PGD \\
Endocytosis & 0.0194 & ARF1, ARPC5, CAV1, DAB2, PRKCI, USP8, \\
Focal adhesion & 0.0368 & CAV1, COL1A2, CTNNB1, FN1 \\
Oxidative phosphorylation & 0.0386 & ATP6VOA4, ATP6V1B2, COX6B1 \\
Synaptic vesicle cycle & 0.0457 & ATP6VOA4, ATP6V1B2 \\
\hline
\end{tabular}

Fig. 13 Meta-analysis of genes reflective for developmental environment and differential expressed between ICM and TE cells. a Venn diagram depicting genes differentially expressed in CVT vs. CVO as well as in ICM vs. TE cells of in vivo derived embryos. The arrows on the left hand indicate expression trend of genes in CVT compared to CVO blastocysts whereas the arrows on the right side indicate expression trend of these genes in ICM relative to TE cells of in vivo derived embryos. $\mathbf{b}$ Venn diagram depicting genes differentially expressed in CVT vs. CVO as well as in ICM vs. TE cells of in vitro derived embryos. The arrows on the left hand indicate expression trend of genes in CVT compared to CVO blastocysts whereas the arrows on the right side indicate expression trend of these genes in ICM relative to TE cells of in vitro derived embryos. c Molecular pathways enriched by genes differentially expressed both in CVT vs. CVO blastocysts as well as in ICM vs. TE cells

increased blood pressure, higher fasting glucose, and increased peripheral body fat deposits in childhood or adolescence in humans $[51,52]$. Therefore, marked transcriptome differences between competent in vivo and in vitro derived blastocysts could partly be associated with postnatal abnormalities in the latter. However, this speculation requires further confirmation by analysing the gene expression outline of blastocyst biopsies ending up with abnormal postnatal consequences. One the other hand, differences in the gene expression patterns may not be stable. Thus, after transfer to recipients, the aberrant expression of these genes might only persist if the adverse environmental conditions persist, otherwise the expression patterns could return to the normal level after transfer to the physiological environment. But, there might be a considerable proportion of genes affected by the environmental condition which do not bear a consequence for an embryo's ability to initiate a robust pregnancy and/or to term development.

Collectively, the results of the present study demonstrated, specific effects of the developmental environment on a bovine embryo's gene expression outline by comparing embryos of comparable developmental capacity. Suboptimal developmental environments, as represented by the in vitro culture in the present study, caused aberrant expression of several gene clusters, especially those coding for ribosomal proteins, mitochondrial ribosomal proteins and NADH dehydrogenases. Noteworthy, especially transcripts related to ribosomal proteins were found to be preferentially expressed in inner cell mass cells compared to trophectoderm cells. Thus, the in vitro environment exhibits profound effects on biological processes associated with metabolism, ATP production and protein synthesis and molecular functions including oxidoreductase and cytochrome-c reductase activity. In the current study, while differentially expressed genes enriched in ribosomal proteins, mitochondrial ribosomal proteins and NADH dehydrogenase were not conflictive with pregnancy establishment at day 90 .

\section{Molecular signatures predictive for embryos' capability to initiate robust pregnancies}

Transferable embryos are usually selected using morphological criteria in cattle as well as humans. Other non-invasive methods include evaluation of morphokinetic features, oxygen consumption and the presence of biochemical molecules within the embryo surrounding spent media [53]. However, non-invasive techniques are not consistent in producing the expected results, as these methods lack direct information about an embryo's intrinsic characteristics. Consequently, embryo selection techniques based on its intrinsic quality using embryo 
biopsies approaches could provide detailed and reliable information about the molecular gene expression signature of the embryos. Therefore, assessing the intrinsic quality of an embryo would provide an alternative option to uncover the molecular mechanism governing successful embryonic development and to select embryos bearing high developmental potential. With this respect, identification of genes showing typical expression patterns reflective of high developmental competency could be an important step to identify molecular markers specifically associated with embryo developmental potential [54]. With this notion, in the current study, we also uncovered the transcriptome profiles of bovine embryos derived either from in vitro or in vivo comparing those with the ability to end up in a robust pregnancy against those lacking the ability to initiate early pregnancy using the EmbryoGENE microarray platform, which consists of about 45,000 probes. Accordingly, the present study identified about 700 and 218 annotated DEGs between competent in vivo derived embryos (CVO) and noncompetent in vivo derived embryos (NVO) as well as competent in vitro derived embryos (CVT) and noncompetent in vitro derived embryos (NVT), respectively. That suggests higher heterogeneities in the transcriptome profile due to developmental competence in embryos derived from in vivo compared to in vitro ones. When investigating the expression trend of differential expressed genes, most (82\%) were upregulated in competent in vivo derived blastocysts compared to the noncompetent ones. In contrast, about $89 \%$ of DEGs were down regulated in the competent in vitro derived embryos compared to their non-competent counterparts. That finding suggests that while increased transcriptional activity in the day 7 in vivo bovine embryos seems to go along with developmental competency, reduced transcriptional activity goes along with developmental competency of in vitro derived embryos. Conversely, higher homogeneity of in vitro derived embryos might be a consequence of higher selection pressure due to less favourable in vitro culture conditions. This assumption, however, is deduced based on overall expression patterns and thus further verification by independent studies is required.

In addition to exploring the global gene expression trends, identification of genes with high expression change and their relevant functions could help to identify transcripts associated with developmental competence. In line to this, 4.4 and $11.4 \%$ of all DEGs exhibited noticeable expression differences $(\geq 3$ fold changes) when comparing competent vs. non-competent in vivo derived embryos and competent vs. noncompetent in vitro derived embryos, respectively (Supplemental Fig. 3). For instance, the expression of ribosomal proteins (RPL34, RPS28, RPS24), keratin 19
(KRT19), myosin light chain 7 (MYL7), glutaredoxin (GLRX), and SERPINE1 mRNA binding protein 1 (SERBP1) was increased by $3.4-6.0$ folds $(p<0.05)$ in competent vs. non-competent in vivo derived embryos (Fig. 2). Although characterizing the role of these genes in embryo development may require further studies, based on the previous findings it can be speculated that these genes could be involved in embryo development by regulating distinct cellular functions. For instance, previous reports have shown the potential role of RPL34, RPS28 and RPS24 in cell proliferation, cell cycle progression, and cell cycle processes [55-57] whereas SERBP1 has been shown to play a role in the regulation of transcription, RNA metabolism and cell proliferation [58]. Therefore, higher expression of RPL34, RPS28, RPS24, $M Y L 7$ and SERBP1 specifically due to higher developmental capacity in embryos derived in vivo, could implicate that the activity of these genes facilitate pregnancy establishment by controlling cell proliferation, cell cycle progression and regulation of transcriptional activities. On the other hand, nanog (NANOG), TNFAIP3 interacting protein 2 (TNIP2), branched chain aminotransferase 2 (BCAT2), FOS like 1, AP-1 transcription factor subunit (FOSL1) and actin beta (ACTB) were among the DEGs whose expression was reduced by $2.3-7.3$ fold in competent compared to non-competent in vivo derived embryos. Among these, NANOG and FOSL1 are believed to be involved in cell linage formation and defects during this critical developmental step could be a main cause of early pregnancy failure and disorders. For instance, higher activity of NANOG, a cell-specific gene and transcription factor related to pluripotency, is believed to be associated with an undifferentiated state of cells and could be involved in the maintenance of pluripotency in a dose-dependent manner [59]. NANOG is expressed in morula and inner cell mass (ICM) cells of human blastocysts whereas its expression is downregulated during mouse implantation [60,61]. Similarly, FOSL1, a gene responsible for the development of trophoblast giant cells is involved in differentiation of embryonic stem (ES) cells to trophoblast lineage-like cells by activation of lineage-specific genes [62]. Lower expression of NANOG and Fosl1 in competent compared to noncompetent in vivo derived embryos could therefore indicate a higher level of cell differentiation in the more competent in vivo derived embryos.

\section{Distinct gene cluster predictive for embryos' capability to initiate robust pregnancies}

In this study, we have identified several gene clusters encompassing groups of genes which potentially share a generalized function, being differentially expressed specifically due to developmental capacity of in vivo derived blastocysts while not observed to be differentially 
regulated in competent vs. non-competent in vitro derived blastocysts. Noteworthy, eukaryotic translation initiation factors such as EIF1, EIF3, EIF3C, EIF3D, EIF3E, EIF3K, EIF4 and EIF4E2 were among these clusters. Within these, the eukaryotic translation initiation factor 3 (eIF3) is the largest complex of the translation initiation factors comprised of thirteen subunits (eIF3a to eIF3m). Mice deficient in eIF3e were embryonically lethal. Depletion of eIF3e is suggested to cause reduced levels of eIF3a and eIF3c subunits, subsequently reducing cellular proliferation, suggesting an important role of eIF3e in embryonic development by affecting the global protein translation [63]. Likewise, the eukaryotic initiation factor 4E (eIF4E) which also showed increased expression in competent in vivo derived embryo within the current study is believed to be involved in mesoderm induction during embryogenesis [64], possibly by regulating cap-dependent translation [65]. Thus, we suggest that higher expression of these eukaryotic translation initiation factors might increase cellular proliferation and differentiation by regulating global protein translation turnover in competent in vivo derived blastocysts.

The ribosomal protein of small and large subunits (RPS3, -6, -8, -21, -24, - 28, RPL7, - 11, - 12, - 13. $-15,-23,-24,-27 \mathrm{~A},-30,-31,-34,-35 \mathrm{~A},-36$, $-37 \mathrm{~A},-38,-39)$ as well as mitochondrial ribosomal proteins (MRPL10, MRPL16, MRPL42, MRPL52, MRPL55 and MRPS18C) were also among the gene clusters increased in competent in vivo derived embryos. Among these, RPS3, $-6,-8,-21,-24$ and RPS28 and large subunits such as RPL11, $-13,-15,-23,-24$, $-27 A,-30,-31,-35,-36,-37 A,-38$ were previously reported to be highly expressed in the human embryos at the blastocyst stage [66]. Bioinformatic analysis showed that these genes are structural constituents of the ribosomes and they are involved in ribosome pathways. Despite the fact that the exact role of ribosomal proteins in embryogenesis is not yet well described, it is well known, however, that small ribosomal proteins play a role in the initiation of translation while the large ribosomal proteins are involved in the formation of peptide bonds [67]. In line with this, a study conducted in HeLa cells indicated that RPS6, RPS8, RPS24 and RPS28 are required for initiation of the processing steps specific to the $18 \mathrm{~S}$ pre-rRNA maturation pathway whereas RPS3 is required for the nuclear and cytoplasmic maturation steps and for nuclear export of proteins [68]. Similarly, other studies also showed the role of RPS6, RPS24, RPS28, RPL7, RPL11 and RPL35A in the production of mature ribosomes and functionally active polysomes [69], and specifically the role RPL11 in embryonic development by regulating p53-dependent checkpoint responses [70]. Thus, increased expression levels of ribosomal proteins in competent in vivo derived blastocysts could reflect a more advanced status of differentiation or functionality in these embryos being beneficial for further development. In addition, further gene clusters associated with energy metabolism were also enriched in competent vs. non-competent in vivo derived blastocysts. These gene clusters include mitochondrial membrane ATP synthases (ATP5) subunits $(E$, G1, G2, $H, I, J, J 2, L, O)$, NADH: ubiquinone oxidoreductase core (NDUF) subunits $(S 1, S 2, S 4, S 5, S 8, B 8)$, cytochrome c oxidase (COX) subunits $(5 A, 6 A 1,17 A 2,6 B 1$, $6 B, 6 C$ ), and aldehyde dehydrogenases (ALDH16A1, $A L D H 3 A 2, A L D H 7 A 1)$. These gene clusters are involved in ATP production, molecular functions including oxidoreductase activity, electron transfer activity, NADH dehydrogenase activity and cytochrome-c oxidase activity specifically indicating the manifestation of higher ATP turnover due to high developmental competence in embryos derived in vivo. Furthermore, literature mining with respect to those individual genes showed that the ATP5E (the $\varepsilon$-Subunit of mitochondrial ATP synthase) gene could be involved in spindle orientation, nuclear divisions and centrosome positioning during embryonic divisions by increasing the ATP synthase activity [71]. Another subunit of ATP5, ATP5H and ATP5B have already been implicated in embryo implantation [72]. Likewise, the NADH: ubiquinone oxidoreductases such as NDUFS1, NDUFS6 and NDUFS 8 are implicated in embryo development whereas cytochrome c oxidase subunits such as COX5A, COX6B1 and COX17 are implicated both in embryonic development and implantation [72]. Collectively, these results strongly indicate that the expression outline of gene clusters associated with mitochondrial functionality might be a good indicator for the capability of the in vivo derived embryo to end up in a robust pregnancy.

To get a more comprehensive overview about the function of these clusters of genes with respect to a blastocysts developmental competence, we analysed in which molecular pathways these clusters are involved. Strikingly, glycolysis, pyruvate metabolism and oxidative phosphorylation molecular pathways were enriched by these genes suggesting higher ATP accumulation in competent in vivo derived embryos using either aerobic or anaerobic energy providing pathways. Indeed, $86 \%$ of ATP production in bovine embryos during blastocyst formation is derived from oxidative phosphorylation [73]. This supports that ATP production of the blastocyst stage embryo is mainly derived from aerobic pathways and thus, higher expression of genes associated with ATP production in competent in vivo derived embryos could be a need for implantation and further development.

Collectively, the present study was able to unravel that compared to the non competent ones; competent in vivo derived embryos are characterized by higher expression 
of gene clusters including those involved in mitochondrial functions, ATP synthases, eukaryotic translation initiation factors, ribosomal proteins as well as $\mathrm{NADH}$ dehydrogenases. This can in turn indicate that competent in vivo derived embryos are characterized by upregulation of global protein translation turnover as well as ATP generating pathways. In contrast, compared to the none competent ones, in vitro derived competent embryos which usually have a low developmental capacity compared to in vivo due to non-physiological developmental environment, showed altered expression of genes involved in protein processing in the endoplasmatic reticulum, splicosome and ubiquitone mediated lysosome functions.

\section{Candidate gene expression signatures predictive for developmental capacity}

Previously, Zoloni et al. [34] have most recently summarized the expression pattern of 19 DEGs related to developmental capacity of in vivo derived bovine embryos reported by Ghanem et al. [33], El-Sayed et al. [31] as well as others [32, 35]. Interestingly, the authors indicated that the expression levels of two candidate genes (EEFA1A and KRT8) were most likely associated with the pregnancy outcome of bovine blastocysts after transfer to recipients. In fact, previous reports [32, 33] were done only using microarray analysis consisting of few numbers genes and other studies [34, 35] were focused on female embryo competency in relation to the pregnancy outcome and embryos were categorized as competent when it induces pregnancy both at day 30 and day 60 while in the current study both in vitro and in vivo embryos were considered as developmentally competent if the pregnancy was maintained until day 90 and beyond. Despite of these differences, we have also merged the DEGs identified in CVO vs. NVO and CVT vs. NVT with that of all previous studies using [31-35] and were also able to identify these two transcripts among those genes being differentially expressed due to contrasting developmental capacity within the present study. Noteworthy, a total of 53 DEGs were commonly detected by our present study as well as by at least one previous one (Table 8). Among these, EEFA1A, KRT8, ZNF281, GART, NMP1, TXN, PAG2G4 and PLAC8 were differentially expressed between competent and noncompetent blastocysts in at least three studies including the current one. Of these, EEFA1A and KRT8 were identified in 5 out of 7 studies suggesting their involvement in pathways being highly correlated with developmental capacity. Moreover, 6 transcripts, namely ZFN281, GART, EEF1A1, HSPA8, RALA and RER1 showed the same trend of expression for competent embryos in all studies analysing in vivo derived ones, whereas 8 transcripts, namely NPM1, ENPEP, TXN, TPT1, RPL26,
H2AFZ, DDX5 and RPA3 showed the same trend of expression for competent in vitro derived embryos in all studies. Finally, 14 transcripts, namely ZNF281, ZNFP36L1, YIPF5, TSMB4X, GART, FERMT2, CYP5A1, CTR9, CDYL, CCRN4L, PRKCQ, PFDN5, CTSZ and $B N I P 3$ showed a similar expression trend both for competent in vivo and in vitro derived embryos. This suggested that these genes could be potentially the reflectors of the intrinsic quality of bovine blastocysts and their developmental competence to establish pregnancy. Conversely, 8 transcripts, namely STAU2, SMAP, SLC36E3, PERP4, CSRP2, RPLP2, DSTN and CLIC1 showed a contradictory expression trend in the competent in vivo and competent in vitro derived embryos, implicating contrasting molecular needs for competent bovine embryos derived from contrasting developmental environments.

\section{Do gene expression signatures reflective for environmental conditions or predictive for developmental capacity resemble gene expression of either ICM or TE cells?}

Developmental competency of a given embryo in general depends on its ability to segregate into two main cell linages, the inner cell mass (ICM) and the trophectoderm (TE). This may lead to the hypothesis that preferential enrichment of genes reflective for developmental capacity in ICM or TE cells may determine the fate of the blastocyst towards establishment of a robust pregnancy. Indeed, previous reports have shown differential expression of genes between ICM and TE cells in mouse [74], human [75] and bovine embryos [42, 43, 76, 77]. Thus, analysis those distinct molecular signatures of the embryo which are predictors of developmental capacity in the cell lineages could be essential to generate basic knowledge to enlighten our understanding about the developmental capacity of bovine embryos. Also, the preferential expression of genes predicting developmental capacity in either ICM or TE cells could give an indication on whether one distinct cell lineage of the blastocyst (ICM or TE) is predominately fraught with problems during early development. Therefore, we superimposed those DEGs identified to be predictive for developmental competence in the current study with comparative transcriptome profiles of the ICM and the TE of in vivo and in vitro derived bovine blastocysts reported by Hosseini et al. [42] and Ozawa et al. [43], respectively. Of high interest, several genes that were differentially expressed between the competent and non-competent in vivo derived embryos were also differentially expressed between the ICM and TE. However, there was no indication for a preferential expression of genes predictive for developmental capacity in either ICM or TE cells considering in vivo and in vitro derived bovine embryos. 
Table 8 Molecular signatures of in vivo and in vitro derived embryos correlated with developmental capacity

\section{In vivo derived embryos}

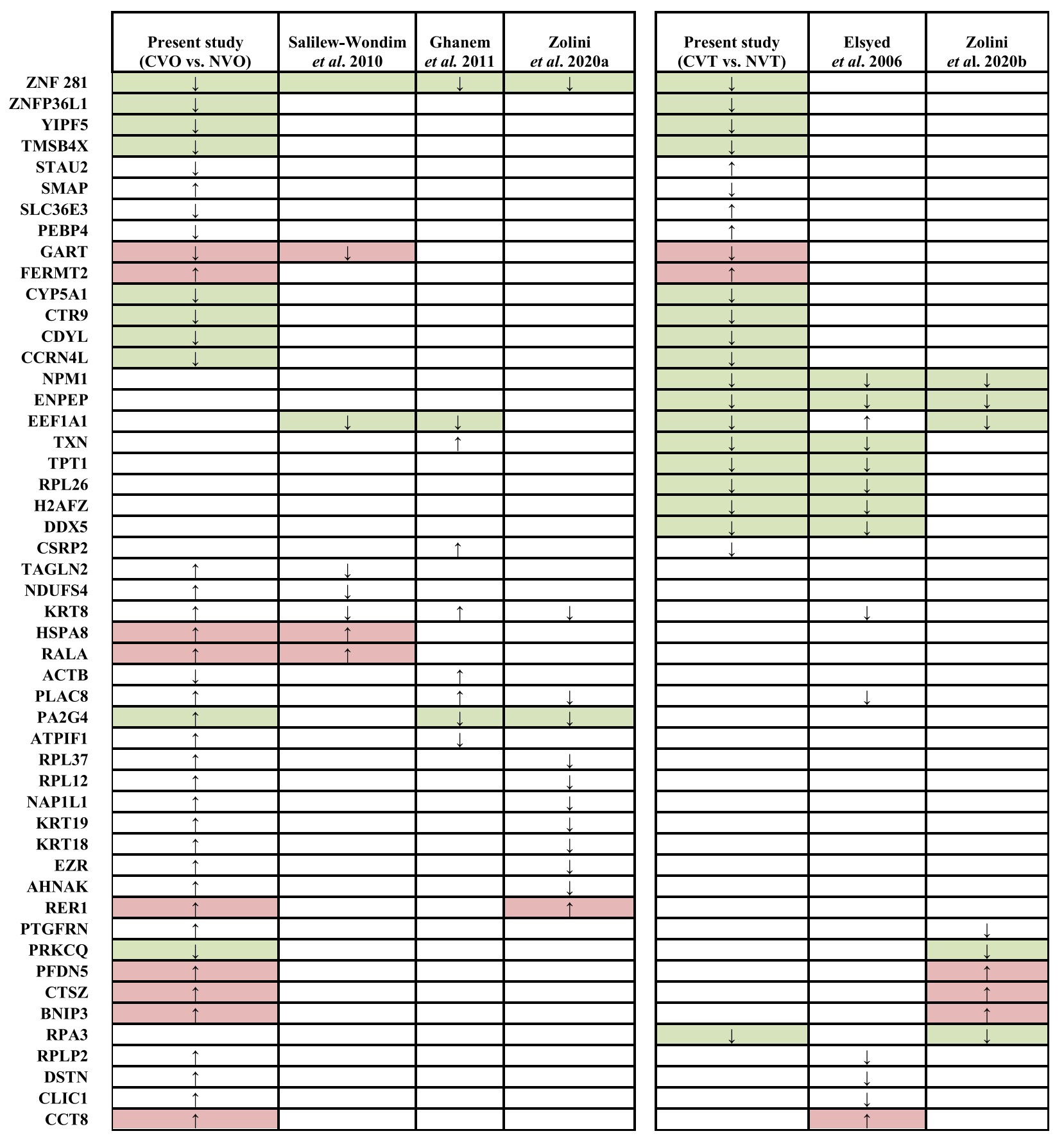

\section{In vitro derived embryos}

Symbols, $\uparrow$ and $\downarrow$ indicate up and downregulated genes in competent blastocysts compared tovnone-competent ones.

Consequently, the present study did not identify one cell lineage prone to developmental problems. In contrast, gene expression signatures specifically correlated with developmental capacity seem to be distributed equally over both compartments.

\section{Conclusion}

In this study, we outlined effects of different developmental environments as well as contrasting developmental capacities of bovine embryos on a gene expression outline. The results of the present study unravelled that 
competent in vivo derived embryos, serving as gold standards, are characterized by higher expression of genes in gene clusters involved in mitochondrial functions, ATP synthases, eukaryotic translation initiation factors and ribosomal proteins, indicating that competent in vivo derived embryos are characterized by upregulation of global protein translation turnover as well as ATP generating pathways. In contrast, in vitro derived embryos of low developmental capacity, showed adverse expression of genes involved in protein processing in the endoplasmatic reticulum, splicosome and ubiquitone mediated lysosome. Since the latter had developed within a non-physiological environment, the present study also demonstrated a specific effect of the developmental environment on a bovine embryo's gene expression outline. To our knowledge, this is the first study in analysing environmental effects on bovine embryos by comparing embryos of comparable developmental capacity instead of using a pool of embryos consisting of different developmental capacity. Representing a main result, non-physiological developmental environments, as represented by the in vitro culture in the present study, cause aberrant expression of gene clusters especially coding for ribosomal proteins, mitochondrial ribosomal proteins and NADH dehydrogenases. Besides, the present study demonstrates that the in vitro environment exhibits down regulative effects on gene signatures, and also shows those gene signatures to be predictive for high developmental capacity of embryos developed in physiological environments. While differential expression of genes enriched in ribosomal proteins, mitochondrial ribosomal proteins and NADH dehydrogenase, as a reflection of non-physiological environments were not conflictive with pregnancy establishment at day 90 ..

Altogether, the present study provides a detailed inventory of differentially expressed candidate genes, gene signatures and pathways reflecting contrasting developmental environments as well as distinct gene signatures and pathways being predictive for future developmental capacity in bovine embryos. Noteworthy, the gene signatures reported to be predictive for developmental capacity by the present study are encompassing gene variants, alternative 3'UTR events and indel type splice variants for the first time. Of high importance, nonphysiological culture environments were found to be reflected in differential expression of gene signatures, which were in turn predictive for low developmental capacities.

\footnotetext{
Abbreviations

CVO: Competent in vivo blastocysts that resulted in pregnancy;

$\mathrm{CVT}$ : Competent in in vitro blastocysts that resulted in pregnancy; NVO: Non competent in vivo blastocysts that resulted in no pregnancy; NVT: Non
}

competent in vitro blastocysts that resulted in no pregnancy; DEGs: Differentially expressed genes

\section{Supplementary Information}

The online version contains supplementary material available at https://doi. org/10.1186/s12864-021-07693-0.

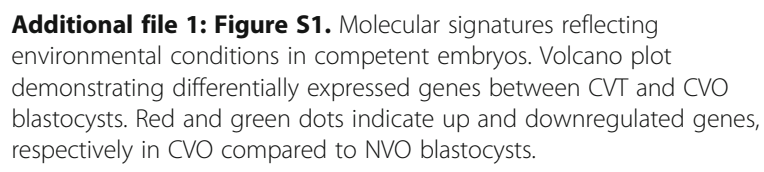

Additional file 2: Figure S2. Molecular pathways significantly enriched by differentially expressed genes specifically modulated by environmental conditions in competent embryos (CVT vs. CVO). Terms on the left represent pathway modules (A) and terms on the right hand represent specific particular pathways enriched by differentially expressed genes (B). NAFLD: Non-alcoholic fatty liver disease.

Additional file 3: Figure S3. Relative proportion of differential expressed genes $(p<0.05)$ within distinct fold change categories in competent vs. non-competent in vivo derived embryos (CVO vs. NVO) and competent vs. non-competent in vitro derived embryos (CVT vs. NVT), respectively.

Additional file 4: Table S1. The list of differentially expressed genes between CVO and NVO. Table S2. The list of differentially expressed genes between CVT and NVT. Table S3. Significant molecular functions enriched by differentially expressed genes between CVT and NVT. Table S4. DEGs common to current study (CVO vs NVO) and Hosseini et al. 2015 (ICM vs .TE) and Ozawa et al. 2012 (TE vs .ICM). Table S5. DEGs common to current study (CVT vs NVT) and Hosseini et al. 2015 (ICM vs .TE) and Ozawa et al. 2012 (TE vs .ICM). Table S6. List of differentially expressed genes between CVT vs. CVO. Table S7. List of differentially expressed probes in CVT vs. CVO representing the 3'-UTR variants. Table S8. Molecular fuctions overrepresented by DEGs idnetfied in CVT vs. CVO. Table S9. Molecular functions overrepresneted by genes differentially expressed between CVT and CVO. Table S10. DEGs common to current study (CVT vs CVO) and Hosseini et al. 2015 (ICM vs .TE) and Ozawa et al. 2012 (TE vs .ICM).

Additional file 5: Table S11. Gene variants differential expressed between CVT and CVO groups.

\section{Acknowledgements}

Not applicable.

\section{Authors' contributions}

DSW: Designed the experiment, performed experiment, performed sample processing and array hybridization, data analysis, interpreting the results, drafting and writing the manuscript. DT: designed the experiment, interpretation of the results and reviewing the manuscript. FR: Sample collection. EH: sample collection. DM: Critically revised the manuscript. MAS: Critically revised the manuscript. ET: Critically revised the manuscript. KS: designed the experiment, supervised the experiments. $\mathrm{MH}$ : Designed the experiment, performed experiment, interpreting the results, reviewing the manuscript and serve as corresponding author. All authors approved the final version of the paper.

\section{Funding}

This study was funded by German Research Foundation (DFG). The funding body has no role in the designing the experiment, collection of data, analysis interpretation of data, writing the manuscript. Open Access funding enabled and organized by Projekt DEAL.

\section{Availability of data and materials}

All data used and/or analyzed during the present study are available from the corresponding author if requested. 


\section{Declarations}

\section{Ethics approval and consent to participate}

The experiment was approved by the Animal Welfare committee of the University of Bonn with proposition number 84-02.05.20.12.075.

\section{Consent for publication}

Not applicable.

\section{Competing interests}

The authors declare that they have no competing interests.

\section{Author details}

${ }^{1}$ Institute of Animal Sciences, Animal Breeding, University of Bonn, Endenicher Allee 15, 53115 Bonn, Germany. ${ }^{2}$ Animal Reproduction and Biotechnology Laboratory, Department of Biomedical Sciences, Colorado State University, 3105 Rampart Rd, CO 80521 Fort Collins, USA. ${ }^{3}$ Centre de Recherche en Reproduction, Développement et Santé Intergénérationnelle, Faculté des sciences de l'agriculture et de l'alimentation, INAF, Pavillon des services, Université Laval (Québec), G1V 0A6, Quebec City, Canada. ${ }^{4}$ Department of Animal Science, Biotechnology \& Reproduction in farm animals, University of Goettingen, Burckhardtweg 2, 37077 Goettingen, Germany.

\section{Received: 19 January 2021 Accepted: 14 April 2021}

\section{Published online: 03 June 2021}

\section{References}

1. Giorgetti C, Terriou P, Auquier P, Hans E, Spach J-L, Salzmann J, et al. Embryo score to predict implantation after in-vitro fertilization: based on 957 single embryo transfers. Hum Reprod. 1995;10(9):2427-31. https://doi. org/10.1093/oxfordjournals.humrep.a136312.

2. Hardarson T, Hanson C, Sjögren A, Lundin K. Human embryos with unevenly sized blastomeres have lower pregnancy and implantation rates: indications for aneuploidy and multinucleation. Hum Reprod. 2001;16(2): 313-8. https://doi.org/10.1093/humrep/16.2.313.

3. Salumets A, Hydén-Granskog C, Mäkinen S, Suikkari A-M, Tiitinen A, Tuuri T. Early cleavage predicts the viability of human embryos in elective single embryo transfer procedures. Hum Reprod. 2003;18(4):821-5. https://doi. org/10.1093/humrep/deg184.

4. van Royen $E$, Mangelschots $K$, Vercruyssen $M$, de Neubourg D, Valkenburg $M$, Ryckaert $G$, et al. Multinucleation in cleavage stage embryos. Hum Reprod. 2003;18(5):1062-9. https://doi.org/10.1093/humrep/deg201.

5. de CSFR, Souza Setti A, Ferreira Braga D PDA, JR IA, JR BE. Blastomere multinucleation: Contributing factors and effects on embryo development and clinical outcome. Hum Fertil (Camb). 2010;13:143-50. https://doi.org/1 0.3109/14647273.2010.508505.

6. Meriano J, Clark C, Cadesky K, Laskin CA. Binucleated and micronucleated blastomeres in embryos derived from human assisted reproduction cycles. Reprod BioMed Online. 2004;9(5):511-20. https://doi.org/10.1016/s1472-6483 (10)61635-5.

7. Yakin K, Balaban B, Urman B. Impact of the presence of one or more multinucleated blastomeres on the developmental potential of the embryo to the blastocyst stage. Fertil Steril. 2005;83(1):243-5. https://doi.org/10.101 6/j.fertnstert.2004.08.016

8. Holte J, Berglund L, Milton K, Garello C, Gennarelli G, Revelli A, et al. Construction of an evidence-based integrated morphology cleavage embryo score for implantation potential of embryos scored and transferred on day 2 after oocyte retrieval. Hum Reprod. 2007;22(2):548-57. https://doi. org/10.1093/humrep/del403.

9. Bó G, Mapletoft R. Evaluation and classification of bovine embryos. Anim Reprod. 2013;54:344-8

10. Rocha JC, Passalia F, Matos FD, Maserati MP, JR, Alves MF, Almeida TG, et al. Methods for assessing the quality of mammalian embryos: how far we are from the gold standard? JBRA Assist Reprod. 2016;20(3):150-8. https://doi. org/10.5935/1518-0557.20160033.

11. Ajduk A, Zernicka-Goetz M. Advances in embryo selection methods. F1000 Biol Rep. 2012:4:11. https://doi.org/10.3410/B4-11.

12. King WA. In: RA MF, editor. Chromosome abnormalities and pregnancy failure in domestic animals: Domestic Animal Cytogenetics: Academic Press; 1990. p. 229-50. https://doi.org/10.1016/B978-0-12-039234-6.50013-6.
13. Yoon S-B, Choi S-A, Sim B-W, Kim J-S, Mun S-E, Jeong P-S, et al. Developmental competence of bovine early embryos depends on the coupled response between oxidative and endoplasmic reticulum stress. Biol Reprod. 2014:90(5):104. https://doi.org/10.1095/biolreprod.113.113480.

14. Yamanaka K-I, Khatun H, Egashira J, Balboula AZ, Tatemoto H, Sakatani M, et al. Heat-shock-induced cathepsin B activity during IVF and culture compromises the developmental competence of bovine embryos. Theriogenology. 2018;114:293-300. https://doi.org/10.1016/j. theriogenology.2018.04.005.

15. Sagirkaya H, Misirlioglu M, Kaya A, First NL, Parrish JJ, Memili E. Developmental and molecular correlates of bovine preimplantation embryos. Reproduction. 2006;131(5):895-904. https://doi.org/10.1530/ rep.1.01021.

16. Purpera MN, Giraldo AM, Ballard CB, Hylan D, Godke RA, Bondioli KR. Effects of culture medium and protein supplementation on mRNA expression of in vitro produced bovine embryos. Mol Reprod Dev. 2009;76(8):783-93. https://doi.org/10.1002/mrd.21028.

17. Kepkova KV, Vodicka P, Toralova T, Lopatarova M, Cech S, Dolezel R, et al. Transcriptomic analysis of in vivo and in vitro produced bovine embryos revealed a developmental change in cullin 1 expression during maternal-toembryonic transition. Theriogenology. 2011;75(9):1582-95. https://doi.org/1 0.1016/j.theriogenology.2010.12.019.

18. Gad A, Hoelker M, Besenfelder U, Havlicek V, Cinar U, Rings F, et al. Molecular mechanisms and pathways involved in bovine embryonic genome activation and their regulation by alternative in vivo and in vitro culture conditions. Biol Reprod. 2012;87(4):100. https://doi.org/10.1095/ biolreprod.112.099697.

19. Badr H, Bongioni G, Abdoon ASS, Kandil O, Puglisi R. Gene expression in the in vitro-produced preimplantation bovine embryos. Zygote. 2007;15(4):35567. https://doi.org/10.1017/S0967199407004315.

20. Mohan M, Hurst AG, Malayer JR. Global gene expression analysis comparing bovine blastocysts flushed on day 7 or produced in vitro. Mol Reprod Dev. 2004;68(3):288-98. https://doi.org/10.1002/mrd.20086.

21. Corcoran D, Fair T, Park S, Rizos D, Patel OV, Smith GW, et al. Suppressed expression of genes involved in transcription and translation in in vitro compared with in vivo cultured bovine embryos. Reproduction. 2006;131(4): 651-60. https://doi.org/10.1530/rep.1.01015.

22. Gutiérrez-Adán A, Rizos D, Fair T, Moreira PN, Pintado B, La Fuente J d, et al. Effect of speed of development on mRNA expression pattern in early bovine embryos cultured in vivo or in vitro. Mol Reprod Dev. 2004;68(4): 441-8. https://doi.org/10.1002/mrd.20113.

23. Lonergan P, Rizos D, Gutierrez-Adán A, Moreira PM, Pintado B, La Fuente de, Boland MP. Temporal divergence in the pattern of messenger RNA expression in bovine embryos cultured from the zygote to blastocyst stage in vitro or in vivo. Biol Reprod 2003;69:1424-1431. doi:https://doi.org/10.1 095/biolreprod.103.018168, 4

24. Hoelker M, Rings F, Lund Q, Ghanem N, Phatsara C, Griese J, et al. Effect of the microenvironment and embryo density on developmental characteristics and gene expression profile of bovine preimplantative embryos cultured in vitro. Reproduction. 2009;137(3):415-25. https:/doi. org/10.1530/REP-08-0370.

25. Salilew-Wondim D, Fournier E, Hoelker M, Saeed-Zidane M, Tholen E, Looft C, et al. Genome-wide DNA methylation patterns of bovine blastocysts developed in vivo from embryos completed different stages of development in vitro. PLoS One. 2015;10(11):e0140467. https://doi.org/10.13 71/journal.pone.0140467.

26. Salilew-Wondim D, Saeed-Zidane M, Hoelker M, Gebremedhn S, Poirier M, Pandey $\mathrm{HO}$, et al. Genome-wide DNA methylation patterns of bovine blastocysts derived from in vivo embryos subjected to in vitro culture before, during or after embryonic genome activation. BMC Genomics. 2018; 19(1):424. https://doi.org/10.1186/s12864-018-4826-3.

27. eSA R, Bruno C, Fleurot R, Daniel N, Archilla C, Peynot N, et al. Alteration of DNA demethylation dynamics by in vitro culture conditions in rabbit preimplantation embryos. Epigenetics. 2012;7:440-6.

28. Urrego R, Bernal-Ulloa SM, Chavarria N, Herrera-Puerta E, Lucas-Hahn A, Herrmann D, et al. Satellite DNA methylation status and expression of selected genes in Bos indicus blastocysts produced in vivo and in vitro. Zygote. 2017;25(2):131-40. https://doi.org/10.1017/S096719941 $600040 \mathrm{X}$

29. Barrera AD, Garcia EV, Hamdi M, Sanchez-Calabuig MJ, Lopez-Cardona AP, Balvis NF, et al. Embryo culture in presence of oviductal fluid induces DNA 
methylation changes in bovine blastocysts. Reproduction. 2017;154(1):1-12. https://doi.org/10.1530/REP-16-0651.

30. Saeed-Zidane M, Tesfaye D, Mohammed Shaker $Y$, Tholen E, Neuhoff $C$, Rings $F$, et al. Hyaluronic acid and epidermal growth factor improved the bovine embryo quality by regulating the DNA methylation and expression patterns of the focal adhesion pathway. PLoS One. 2019;14(10):e0223753. https://doi.org/10.1371/journal.pone.0223753.

31. El-Sayed A, Hoelker M, Rings F, Salilew D, Jennen D, Tholen E, et al. Largescale transcriptional analysis of bovine embryo biopsies in relation to pregnancy success after transfer to recipients. Physiol Genomics. 2006;28(1): 84-96. https://doi.org/10.1152/physiolgenomics.00111.2006.

32. Salilew-Wondim D, Holker M, Rings F, Ghanem N, Ulas-Cinar M, Peippo J, et al. Bovine pretransfer endometrium and embryo transcriptome fingerprints as predictors of pregnancy success after embryo transfer. Physiol Genomics. 2010;42(2):201-18. https://doi.org/10.1152/ physiolgenomics.00047.2010.

33. Ghanem N, Salilew-Wondim D, Gad A, Tesfaye D, Phatsara C, Tholen E, et al. Bovine blastocysts with developmental competence to term share similar expression of developmentally important genes although derived from different culture environments. Reproduction. 2011;142(4):551-64. https:// doi.org/10.1530/REP-10-0476.

34. Zolini AM, Block J, Rabaglino MB, Rincon G, Hoelker M, Bromfield JJ, et al. Genes associated with survival of female bovine blastocysts produced in vivo. Cell Tissue Res. 2020;382(3):665-78. https://doi.org/10.1007/s00441020-03257-y.

35. Zolini AM, Block J, Rabaglino MB, Tríbulo P, Hoelker M, Rincon G, et al. Molecular fingerprint of female bovine embryos produced in vitro with high competence to establish and maintain pregnancyt. Biol Reprod. 2020; 102(2):292-305. https://doi.org/10.1093/biolre/ioz190.

36. Ritchie ME, Silver J, Oshlack A, Holmes M, Diyagama D, Holloway A, et al. A comparison of background correction methods for two-colour microarrays. Bioinformatics. 2007;23(20):2700-7. https://doi.org/10.1093/bioinformatics/ btm412.

37. Smyth GK, Speed T. Normalization of CDNA microarray data. Methods. 2003; 31(4):265-73. https://doi.org/10.1016/s1046-2023(03)00155-5.

38. Yang YH, Dudoit S, Luu P, Lin DM, Peng V, Ngai J, et al. Normalization for CDNA microarray data: a robust composite method addressing single and multiple slide systematic variation. Nucleic Acids Res. 2002;30(4):e15-115. https://doi.org/10.1093/nar/30.4.e15.

39. Smyth GK. Limma: linear models for microarray data. In: gentleman R, Carey VJ, Huber W, Irizarry Ra, Dudoit S, editors. bioinformatics and computational biology solutions using $\mathrm{R}$ and bioconductor. Statistics for biology and health. New York: Springer; 2005

40. Robert C, Nieminen J, Dufort I, Gagne D, Grant JR, Cagnone G, et al. Combining resources to obtain a comprehensive survey of the bovine embryo transcriptome through deep sequencing and microarrays. Mol Reprod Dev. 2011;78(9):651-64. https://doi.org/10.1002/mrd.21364.

41. Chen L, Wang D, Wu Z, Ma L, Daley GQ. Molecular basis of the first cell fate determination in mouse embryogenesis. Cell Res. 2010;20(9):982-93. https:// doi.org/10.1038/cr.2010.106.

42. Hosseini SM, Dufort I, Caballero J, Moulavi F, Ghanaei HR, Sirard MA. Transcriptome profiling of bovine inner cell mass and trophectoderm derived from in vivo generated blastocysts. BMC Dev Biol. 2015;15(1):49. https://doi.org/10.1186/s12861-015-0096-3.

43. Ozawa M, Sakatani M, Yao J, Shanker S, Yu F, Yamashita R, et al. Global gene expression of the inner cell mass and trophectoderm of the bovine blastocyst. BMC Dev Biol. 2012;12(1):33. https://doi.org/10.1186/1471-213 $\mathrm{X}-12-33$.

44. Gunesdogan U, Surani MA. Developmental competence for primordial germ cell fate. Curr Top Dev Biol. 2016;117:471-96. https://doi.org/10.1016/bs. ctdb.2015.11.007.

45. Braude $\mathrm{P}$, Bolton V, Moore S. Human gene expression first occurs between the four- and eight-cell stages of preimplantation development. Nature (London). 1988;332(6163):459-61. https://doi.org/10.1038/332459a0.

46. Niakan KK, Han J, Pedersen RA, Simon C, Pera RA. Human pre-implantation embryo development. Development. 2012;139(5):829-41. https://doi.org/1 $0.1242 /$ dev. 060426 .

47. Schultz RM. Regulation of zygotic gene activation in the mouse. Bioessays. 1993;15(8):531-8. https://doi.org/10.1002/bies.950150806.

48. Frei RE, Schultz GA, Church RB. Qualitative and quantitative changes in protein synthesis occur at the 8-16-cell stage of embryogenesis in the cow. J Reprod Fertil. 1989;86(2):637-41. https://doi.org/10.1530/jff.0. 0860637.

49. Wang S, Cowan CA, Chipperfield H, Powers RD. Gene expression in the preimplantation embryo: in-vitro developmental changes. Reprod BioMed Online. 2005;10(5):607-16. https://doi.org/10.1016/s1472-6483 (10)61668-9.

50. Ealy AD, Wooldridge LK, McCoski SR, BOARD INVITED REVIEW. Post-transfer consequences of in vitro-produced embryos in cattle. J Anim Sci. 2019;97(6): 2555-68. https://doi.org/10.1093/jas/skz116.

51. Duranthon V, Chavatte-Palmer P. Long term effects of ART: what do animals tell us? Mol Reprod Dev. 2018;85(4):348-68. https://doi.org/10.1 002/mrd.22970

52. Bonilla L, Block J, Denicol AC, Hansen PJ. Consequences of transfer of an in vitro-produced embryo for the dam and resultant calf. J Dairy Sci. 2014; 97(1):229-39. https://doi.org/10.3168/jds.2013-6943.

53. Sallam HN, Sallam NH, Sallam SH. Non-invasive methods for embryo selection. Facts Views Vis Obgyn. 2016;8(2):87-100.

54. Taylor J, Fairburn H, Beaujean N, Meehan R, Young L. Gene expression in the developing embryo and fetus. Reprod Suppl. 2003;61:151-65.

55. Kim HK, Xu J, Chu K, Park H, Jang H, Li P, et al. A tRNA-Derived Small RNA Regulates Ribosomal Protein S28 Protein Levels after Translation Initiation in Humans and Mice. Cell Rep. 2019;29:3816-3824.e4. https://doi.org/10.1016/j. celrep.2019.11.062.

56. Song B, Zhang Q, Zhang Z, Wan Y, Jia Q, Wang X, et al. Systematic transcriptome analysis of the zebrafish model of diamond-blackfan anemia induced by RPS24 deficiency. BMC Genomics. 2014;15(1):759. https://doi. org/10.1186/1471-2164-15-759.

57. Liu H, Liang S, Yang X, Ji Z, Zhao W, Ye X, et al. RNAi-mediated RPL34 knockdown suppresses the growth of human gastric cancer cells. Oncol Rep. 2015;34(5):2267-72. https://doi.org/10.3892/or.2015.4219.

58. Ahn JW, Kim S, Na W, Baek SJ, Kim JH, Min K, et al. SERBP1 affects homologous recombination-mediated DNA repair by regulation of CtIP translation during S phase. Nucleic Acids Res. 2015;43(13):6321-33. https:// doi.org/10.1093/nar/gkv592.

59. Hatano SY, Tada M, Kimura H, Yamaguchi S, Kono T, Nakano T, et al. Pluripotential competence of cells associated with Nanog activity. Mech Dev. 2005;122(1):67-79. https://doi.org/10.1016/j.mod.2004.08.008.

60. Chambers I, Colby D, Robertson M, Nichols J, Lee S, Tweedie S, et al. Functional expression cloning of Nanog, a pluripotency sustaining factor in embryonic stem cells. Cell. 2003;113(5):643-55. https://doi.org/10.1016/ S0092-8674(03)00392-1.

61. Hambiliki F, Strom S, Zhang P, Stavreus-Evers A. Co-localization of NANOG and OCT4 in human pre-implantation embryos and in human embryonic stem cells. J Assist Reprod Genet. 2012;29(10):1021-8. https://doi.org/10.1 007/s10815-012-9824-9.

62. Lee BK, Uprety N, Jang YJ, Tucker SK, Rhee C, LeBlanc L, et al. Fosl1 overexpression directly activates trophoblast-specific gene expression programs in embryonic stem cells. Stem Cell Res. 2018;26:95-102. https:// doi.org/10.1016/j.scr.2017.12.004

63. Sadato D, Ono T, Gotoh-Saito S, Kajiwara N, Nomura N, Ukaji M, et al. Eukaryotic translation initiation factor 3 (elF3) subunit e is essential for embryonic development and cell proliferation. FEBS Open Bio Open Access. 2018:8(8):1188-201. https://doi.org/10.1002/2211-5463.12482.

64. Klein PS, Melton DA. Induction of mesoderm in Xenopus laevis embryos by translation initiation factor 4E. Science (Washington D C). 1994;265(5173): 803-6. https://doi.org/10.1126/science.8047887.

65. Sonenberg N, Gingras AC. The mRNA $5^{\prime}$ cap-binding protein elF4E and control of cell growth. Curr Opin Cell Biol. 1998;10(2):268-75. https://doi. org/10.1016/s0955-0674(98)80150-6.

66. Kakourou G, Jaroudi S, Tulay P, Heath C, Serhal P, Harper JC, et al. Investigation of gene expression profiles before and after embryonic genome activation and assessment of functional pathways at the human metaphase II oocyte and blastocyst stage. Fertil Steril. 2013;99:803-814.e23.

67. Gregory B, Rahman N, Bommakanti A, Shamsuzzaman M, Thapa M, Lescure A, et al. The small and large ribosomal subunits depend on each other for stability and accumulation. Life Sci Alliance. 2019. https://doi.org/10.26508/ Isa.201800150.

68. O'Donohue MF, Choesmel V, Faubladier M, Fichant G, Gleizes PE. Functional dichotomy of ribosomal proteins during the synthesis of mammalian $40 \mathrm{~S}$ ribosomal subunits. J Cell Biol. 2010;190(5):853-66. https://doi.org/10.1083/ jcb.201005117. 
69. Robledo S, Idol RA, Crimmins DL, Ladenson JH, Mason PJ, Bessler M. The role of human ribosomal proteins in the maturation of rRNA and ribosome production. RNA. 2008;14(9):1918-29. https://doi.org/10.1261/rna.1132008.

70. Chakraborty A, Uechi T, Higa S, Torihara H, Kenmochi N. Loss of ribosomal protein L11 affects zebrafish embryonic development through a p53-dependent apoptotic response. PLoS One [Electronic Resource]. 2009;4:e4152.

71. Kidd T, Abu-Shumays R, Katzen A, Sisson JC, Jiménez G, Pinchin S, et al. The epsilon-subunit of mitochondrial ATP synthase is required for normal spindle orientation during the Drosophila embryonic divisions. Genetics. 2005;170(2):697-708. https://doi.org/10.1534/genetics.104.037648.

72. Fu Z, Wang B, Wang S, Wu W, Wang Q, Chen Y, et al. Integral proteomic analysis of blastocysts reveals key molecular machinery governing embryonic diapause and reactivation for implantation in Mice1. Biol Reprod. 2014;90(3):52. https://doi.org/10.1095/biolreprod.113.115337.

73. Thompson JG, Partridge RJ, Houghton FD, Cox Cl, Leese HJ. Oxygen uptake and carbohydrate metabolism by in vitro derived bovine embryos. J Reprod Fertil. 1996;106(2):299-306. https://doi.org/10.1530/jff.0.1060299.

74. Giritharan G, Delle Piane L, Donjacour A, Esteban FJ, Horcajadas JA, Maltepe $E$, et al. In vitro culture of mouse embryos reduces differential gene expression between inner cell mass and trophectoderm. Reprod Sci. 2012; 19(3):243-52. https://doi.org/10.1177/1933719111428522.

75. Reijo Pera RA, DeJonge C, Bossert N, Yao M, Hwa Yang JY, Asadi NB, et al. Gene expression profiles of human inner cell mass cells and embryonic stem cells. Differentiation. 2009;78(1):18-23. https://doi.org/10.1016/j.diff.2 009.03.004.

76. Fujii T, Moriyasu S, Hirayama H, Hashizume T, Sawai K. Aberrant expression patterns of genes involved in segregation of inner cell mass and trophectoderm lineages in bovine embryos derived from somatic cell nuclear transfer. Cell Rep. 2010;12(5):617-25. https://doi.org/10.1089/cell.201 0.0017 .

77. Zhao X-M, Cui L-S, Hao H-S, Wang H-Y, Zhao S-J, Du W-H, et al. Transcriptome analyses of inner cell mass and trophectoderm cells isolated by magnetic-activated cell sorting from bovine blastocysts using single cell RNA-seq. Reprod Domest Anim. 2016;51(5):726-35. https://doi.org/10.1111/ rda. 12737

\section{Publisher's Note}

Springer Nature remains neutral with regard to jurisdictional claims in published maps and institutional affiliations.

Ready to submit your research? Choose BMC and benefit from:

- fast, convenient online submission

- thorough peer review by experienced researchers in your field

- rapid publication on acceptance

- support for research data, including large and complex data types

- gold Open Access which fosters wider collaboration and increased citations

- maximum visibility for your research: over $100 \mathrm{M}$ website views per year

At $\mathrm{BMC}$, research is always in progress.

Learn more biomedcentral.com/submissions 